Cahiers $d u$ MONDE RUSSE

\section{Cahiers du monde russe}

Russie - Empire russe - Union soviétique et États indépendants

45/3-4 | 2004

Varia

\title{
Hordes faméliques et colons militaires en Russie d'après le baron de la Ruë (1834)
}

Édith Ybert-Chabrier

\section{OpenEdition}

Journals

Édition électronique

URL : https://journals.openedition.org/monderusse/8699

DOI : 10.4000/monderusse.8699

ISSN : $1777-5388$

Éditeur

Éditions de l'EHESS

Édition imprimée

Date de publication : 1 juillet 2004

Pagination : $521-560$

ISBN : 2-7132-2009-2

ISSN : $1252-6576$

Référence électronique

Édith Ybert-Chabrier, « Hordes faméliques et colons militaires en Russie d'après le baron de la Ruë (1834) ", Cahiers du monde russe [En ligne], 45/3-4 | 2004, mis en ligne le 01 janvier 2007, consulté le 04 septembre 2022. URL : http://journals.openedition.org/monderusse/8699; DOI : https://doi.org/ 10.4000/monderusse. 8699 
chercher : repérer : avancer

Cet article est disponible en ligne à l'adresse :

http://www.cairn.info/article.php?ID REVUE=CMR\&ID NUMPUBLIE=CMR 453\&ID ARTICLE=CMR 4530521

Hordes faméliques et colons militaires en Russie d'après le baron de la Ruë (1834)

par ÉDITH YBERT-CHABRIER

Editions de I'EHESS | Cahiers du monde russe

2004/3-4 - Vol 45

ISSN 1252-6576 | ISBN 2713220092 | pages 521 à 560

Pour citer cet article :

-YBERT-CHABRIER ., Hordes faméliques et colons militaires en Russie d'après le baron de la Ruë (1834), Cahiers du monde russe 2004/ 3-4, Vol 45, p. 521-560.

Distribution électronique Cairn pour les Editions de l'EHESS.

(C) Editions de l'EHESS. Tous droits réservés pour tous pays.

La reproduction ou représentation de cet article, notamment par photocopie, n'est autorisée que dans les limites des conditions générales d'utilisation du site ou, le cas échéant, des conditions générales de la licence souscrite par votre établissement. Toute autre reproduction ou représentation, en tout ou partie, sous quelque forme et de quelque manière que ce soit, est interdite sauf accord préalable et écrit de l'éditeur, en dehors des cas prévus par la législation en vigueur en France. Il est précisé que son stockage dans une base de données est également interdit. 


\title{
HORDES FAMÉLIQUES ET COLONS MILITAIRES EN RUSSIE D'APRÈS LE BARON DE LA RUË (1834)
}

\begin{abstract}
Aide de camp du maréchal Maison, ambassadeur de France à Saint-Pétersbourg, Aristide, Isidore, Jean, Marie de La Ruë ${ }^{1}$ est envoyé en mission dans les gouvernements méridionaux de la Russie où il séjourne en avril et mai 1834 avant de se rendre à Constantinople puis dans les principautés de Moldavie et de Valachie et de regagner les rives de la Néva. Au cours de cette mission, il rédige sept rapports portant sur la flotte et les ports militaires de la mer Noire, l'état agricole des gouvernements du Midi, le Caucase et les provinces au-delà, son séjour à Constantinople, les principautés de Valachie et de Moldavie, les colonies militaires, et enfin sur l'état de l'opinion publique en Russie. Conservés aux Archives nationales et au Quai d'Orsay², ces rapports ont été transcrits par George Ciorănescu et transmis par son épouse, auxquels nous exprimons notre profonde reconnaissance. G. Ciorănescu a publié, quelques années avant sa mort, survenue en 1993, une présentation des séjours du baron de La Ruë à Constantinople et dans les provinces roumaines ${ }^{3}$. Ce
\end{abstract}

1. A. I. J. M. de La Ruë (1795-1872), officier de cavalerie, aide de camp du maréchal Marmont, duc de Raguse, de 1815 au début de 1823 , puis, après l'expédition d'Espagne à laquelle il participe, de la fin de 1823 à 1830. Étant resté fidèlement au service du duc de Raguse, même lorsque ce dernier refuse d'abandonner Charles X en juillet 1830, il doit prendre des congés de disponibilité. Il reçoit une nouvelle affectation grâce au maréchal Maison qui intervient en sa faveur et le demande pour aide de camp à Vienne (1832) puis à Saint-Pétersbourg (décembre 1833-mars 1835). Il accomplit à partir de 1836 de nombreuses missions en Afrique du Nord et devient directeur des affaires de l'Algérie au ministère de la Guerre (1845-1848). Promu général en 1849, il finit sa carrière comme président du comité de la gendarmerie.

2. Aux Archives nationales: originaux manuscrits dans Archives Maison, AP 156/III/19, carton 3. Aux Archives du ministère des Affaires étrangères (AMAE), copies des rapports de La Ruë dans Mémoires et documents, vol. 37, f. 85-159.

3. Georges Cioranesco, « Le séjour à Constantinople du baron de La Rue (1834) », in L'Empire ottoman, la république de Turquie et la France, sous la dir. de Hâmit Batu et Jean-Louis Bacqué-Grammont, Istanbul, Éditions Isis, 1986, p. 169-178; George Cioranescu, «Le passage du baron de La Rue par les principautés roumaines (1834) », in Von der Pruth-Ebene 
sont les rapports sur l'état agricole dans les gouvernements du Midi, en fait une description saisissante de la famine de 1833-1834, et sur les colonies militaires que nous présentons ici.

Ces deux textes se complètent et se croisent même en Nouvelle Russie (actuelle Ukraine méridionale), dont les colonies militaires ont honorablement traversé la période de disette. Ils n'en sont pas moins éminemment différents. Dans le premier, La Ruë dresse un tableau - peint sur le vif ou restituant l'émotion de ses informateurs - des populations décimées par la famine dans de nombreux gouvernements et errant sur les routes à la recherche de contrées moins sinistrées. Il décrit aussi les mesures prises pour lutter contre la famine et la représentation qu'il donne a le grand mérite de lier entre eux toutes sortes de faits que les études statistiques ou thématiques présentent séparément. Quant au rapport sur les colonies militaires, il s'intéresse à une institution créée par Alexandre Ier en 1809, développée à grande échelle à partir de 1816 et atteignant son expansion maximale sous son successeur, Nicolas Ir. Il en expose les buts, les réalisations dans la région de Novgorod et dans le Sud et en évalue les résultats tant du point de vue économique que militaire. Le texte reprend un article paru dans Le Spectateur militaire en 1828 et le complète lorsque des faits nouveaux sont survenus. Il traite des questions débattues dans un certain nombre d'articles ou de livres parus en France sur ce sujet qui, selon les mots d'un auteur militaire contemporain, « a pour nous en ce moment deux sortes d'intérêt, la nouveauté et la peur $»^{4}$.

C'est bien cette crainte inspirée par la puissance militaire de la Russie et par ses succès récents sur l'Empire ottoman et sur la Perse qui pousse le maréchal Maison à envoyer le baron de La Ruë en mission dans le Sud de la Russie. Comme il l'écrit, le 31 mars 1834, au duc de Broglie, ministre des Affaires étrangères de LouisPhilippe, il est en train de préparer une étude précise et complète sur l'organisation militaire et l'état des forces en Russie 5 . Pour éviter les erreurs, il souhaite un contrôle minutieux des informations qu'il a pu recueillir. Et, poursuit-il :

À cet effet, Monsieur le duc, j'ai jugé convenable de confier à mon aide de camp, $\mathrm{M}^{\mathrm{r}}$ le baron de la Ruë la mission de parcourir les provinces méridionales de la Russie. [...] J'ai saisi le prétexte du désir qu'avait précédemment témoigné $\mathrm{M}^{\mathrm{r}}$ de la Ruë de visiter Constantinople et le Bosphore, et tout en paraissant condescendre à ses vœux personnels, je m'assure le moyen de réaliser un projet dont nul autre ne pourrait s'acquitter avec autant de chances de succès. J'en ai pour garanties les connaissances spéciales, l'esprit d'observation et les qualités de tout genre qui distinguent le Baron de La Ruë. En même temps, $M^{r}$ de la Ruë recevra pour instruction d'observer la nature des préparatifs qui, malgré les dénégations réitérées du $\mathrm{C}^{\text {te }}$ de Nesselrode, paraissent avoir lieu sur le côtes de la

bis zum Gipfel des Ida, Fetschrift zum 70. Geburtstag von Emanuel Turczynski, Munich, Südosteuropa-Gesellschaft, 1989, p. 185-191.

4. C. J. Ferry, «Observations sur les résultats probables de l'établissement des colonies », in R. Lyall, Notice sur l'organisation, l'administration et l'état des colonies militaires de la Russie, Paris, 1825, p. 57.

5. Cette étude se trouve aux Archives nationales dans les Archives Maison, AP 156/III/ 19, carton 18. 
mer Noire ${ }^{6}$. Il pourra me rapporter également des notions précises sur la situation en Perse et en Géorgie ainsi que sur l'avenir agricole des provinces centrales qu'a dévastées la famine ; et son voyage coïncidant avec l'époque pressentie de l'évacuation des Principautés, je le charge d'en suivre les mouvements $[\ldots]^{7}$.

Maison a rédigé une série de questions qu'il remet à $\mathrm{M}^{\mathrm{r}}$ de la Ruë. Elles portent sur la marine, l'armée, le Caucase, la Perse, l'intérieur agricole, la Turquie et les Principautés, la Bessarabie. Celles qui ont trait à l'intérieur agricole se présentent sous cette forme :

Recueillir les données positives sur l'avenir des Provinces méridionales et surtout des gouvernements de Kharkoff, Voronèje, Koursk, Sloboda d'Ukraine, Ekaterinoslaff et Kherson. Les semailles ont-elles en effet manqué dans divers districts de ces gouvernements ? A-t-on pris des mesures pour remédier à la mortalité des bestiaux ?

Quelle est l'opinion comparée du littoral et de l'intérieur sur la libre importation future des céréales ? Y est-on opposé à Koursk et à Kharkoff ?

Il sera bon de rechercher si, dans la plupart des localités envahies par la disette, l'administration civile s'est montrée imprévoyante et nulle tandis que l'autorité militaire avait presque partout par la sagesse de ses prévisions assuré le service et garanti les intérêts de l'État ${ }^{8}$.

\section{La famine de 1833-1834}

S'agit-il de l'une de ces mauvaises récoltes qui frappe de temps à autre telle ou telle région du vaste Empire russe ou d'une famine exceptionnelle ? Les disettes jalonnent en effet l'histoire russe. Slovcov, dans l'étude qu'il leur consacre, en dénombre cent trente de 1024 à $1854^{\circ}$. Mais certaines ne touchent que quelques gouvernements, alors que d'autres, plus sévères, frappent de vastes zones. Le $\mathrm{XIX}^{\mathrm{e}}$ siècle a connu trois famines particulièrement dévastatrices, celles de 1833$1834,1872-1873$ et $1891-1892^{10}$. À partir des années 1820 , les régions méridionales ont souffert d'une succession de mauvaises récoltes :

1823, dans le gouvernement de Kherson, d'Ekaterinoslav et de Tauride ;

6. La France et l'Angleterre sont particulièrement inquiètes des avancées russes en Orient : en juillet 1833, s'étant porté au secours de la Porte menacée par le vice-roi d'Égypte, MéhémetAli, la Russie a conclu avec cette dernière le traité défensif d'Unkiar-Skelessi, lui assurant une position très avantageuse en mer Noire grâce à un accord sur les Détroits. D'où une surveillance du moindre mouvement de navire russe en partance de la Crimée et des doutes sur les déclarations du vice-chancelier chargé des Affaires étrangères de l'Empire russe, le $\mathrm{C}^{\text {te }}$ de Nesselrode.

7. AMAE, Correspondance politique Russie, vol. 188, f. 172.

8. Ibid., f. 174-175

9. Slovcov, «Istoričeskoe i statističeskoe obozrenie neuroažev v Rossii » (Étude historique et statistique des disettes en Russie), Sbornik statističeskih svedenij o Rossii izdavaemyj statističeskim otdeleniem imperatorskogo russkogo geografičeskogo ob̌̌čestva, t. III, SaintPétersbourg, 1858, p. 466-473.

10. V. V. Kondrašin, «Golod v krest’ janskom mentalitete» (La famine dans la mentalité paysanne), in Mentalitet i agrarnoe razvitie Rossii, $X I X-X X V V$. (Mentalité et développement agraire en Russie, $X I X-X X^{e}$ siècle), Moscou, 1996, p. 115. 
1824, en Nouvelle Russie, en fait dans les mêmes gouvernements que l'année précédente ;

1827, dans la Russie méridionale et particulièrement dans le gouvernement de Tauride (actuelle Crimée) ;

1833, en Nouvelle Russie et dans le gouvernement de Tchernigov ;

1834, dans les gouvernements de Vitebsk, de Volhynie, de Koursk, de Pskov, de Kherson, Orlov, Poltava, Tambov, Riazan, Tchernigov et en Bessarabie.

Cette description de Slovcov ${ }^{11}$ peut induire en erreur : l'année noire a bien été 1833, mais les effets du manque absolu de récoltes se font encore sentir l'année suivante dans de nombreuses régions, où sévit toujours la faim et où les semailles sont parfois restreintes. En 1834, les récoltes sont dans l'ensemble bonnes sauf dans quelques gouvernements dont effectivement ceux de Riazan et de Tambov touchés par la disette $^{12}$. En 1833, la moitié de la Russie d'Europe, c'est-à-dire, les gouvernements au sud du $53^{\circ}$ de latitude, peuplée de 14,5 millions d'habitants, a vu ses récoltes de céréales anéanties ${ }^{13}$. La production de grains, pour l'ensemble de la Russie, a baissé de $32 \%$, alors que, d'après les données annuelles collectées depuis le début du XIX ${ }^{\mathrm{e}}$ siècle, dans la plupart des disettes, cette baisse représente environ $10 \%{ }^{14}$.

Ayant constaté l'ampleur de cette famine, voyons le nombre de morts qu'elle a provoquées. Bien que, dans les années 1830, les statistiques démographiques russes deviennent plus précises et fiables, il n'y a pas de dénombrement général des victimes de la famine de 1833, mais il est possible de se faire une idée approximative de leur nombre grâce aux chiffres donnés par Roslavskij. Travaillant sur les documents établis par l'Église orthodoxe, qui chaque année enregistre et déclare naissances, mariages et décès de ses ressortissants, l'auteur se limite donc, pour ses calculs, à la seule population orthodoxe. Les tableaux qu'il présente font apparaître deux pics de mortalité dans les années 1825-1837 : 1831, année durant laquelle l'épidémie de choléra fait de très nombreuses victimes, avec 1621000 morts, et 1833, année marquée par la famine, avec 1545291 morts. Pour avoir une idée approximative du nombre de morts supplémentaires, imputables à la famine, comparons la mortalité de 1833 à celles des années proches qui ne sont pas marquées par des calamités exceptionnelles : 1830, 1832, 1834, 1835, 1836, 1837. Ainsi, il est

\section{Slovcov, art. cit., p 471-472.}

12. I. D. Koval`čenko, « $\mathrm{K}$ voprosu ob urovne sel'skohozjajstvennogo proizvodstva $\mathrm{v}$ Rjazanskoj i Tambovskoj gubernijah v konce XVIII-pervoj polovine XIX veka » (La question du niveau de la production agricole dans les gouvernements de Riazan et de Tambov à la fin du $\mathrm{XVIII}^{\mathrm{e}}$ siècle et dans la première moitié du XIX ${ }^{\mathrm{e}}$ siècle), in Materjaly po istorii sel'skogo hozjajstva i krest'janstva SSSR (Matériaux pour l'histoire de l'agriculture et de la paysannerie en URSS), vol III, Moscou, 1959, p. 259-260.

13. V. I. Pokrovskij, «Vlijanie kolebanij urožaja i hlebnyh cen na estestvennoe dviženie naselenija » (Influence des variations des récoltes et des prix des céréales sur le mouvement naturel de population), in Vlijanie urožaev i hlebnyh cen na nekotorye storony russkogo narodnogo hozjajstva (Influence des récoltes et des prix des céréales sur quelques aspects de l'économie russe), Saint-Pétersbourg, 1897, p. 186.

14. A. V. Dulov, Geografičeskaja sreda i istorija Rossii. Konec XV-seredina XIX V. (Le milieu géographique et l'histoire de la Russie. Fin XVe-milieu du XIX ${ }^{e}$ s.), Moscou, 1983, p. 52-53. 
possible de déduire qu'un peu plus de 200000 morts seraient imputables à la famine. Ses victimes sont particulièrement nombreuses chez les nourrissons et les enfants, et dans une moindre mesure chez les jeunes de moins de trente ans, à la différence du choléra qui a d'avantage décimé les gens âgés de 45 à 60 ans ${ }^{15}$.

Quant aux mesures prises pour lutter contre la famine, La Ruë en passe bon nombre en revue. Est-il impartial on systématiquement négatif ? Il remplit sans doute au mieux la mission d'information qui lui est confiée et restitue de nombreuses critiques qu'il a entendues sur l'application des mesures prises. Il nous semble cependant, que n'ayant pas une idée précise de l'organisation générale du système d'approvisionnement de la Russie, il lui manque un élément important d'appréciation. Comme beaucoup de ses contemporains qui s'intéressent à la Russie, il ne conçoit pas que ce pays ait à la fois un gouvernement autocratique et des institutions sociales reposant sur des bases juridiques. Aveuglés par son despotisme et par sa pauvreté, ils la conçoivent comme un État sauvage où la loi a peu de place, sous-estimant la rationalisation des institutions économiques et sociales en cours ainsi que l'emprise de l'homme sur la nature. Il me semble donc important, pour un bon éclairage du rapport sur l'état agricole des gouvernements du Midi reproduit ici, de rappeler les grandes lignes du système d'approvisionnement prévu pour faire face aux mauvaises récoltes en Russie.

Nourrir les paysans et les citadins les années de disette, leur donner assez de subsistance pour les maintenir en vie sans qu'ils aient à partir mendier sur les grands chemins, fait traditionnellement partie des devoirs de leurs autorités de tutelle : propriétaires terriens pour les serfs, l'État par l'intermédiaire de ses représentants pour les paysans de la Couronne ou les habitants des villes. Pour ce faire, diverses lois ont été adoptées, surtout depuis le règne de Pierre le Grand afin d'éviter les famines. Leur application, toujours partielle, n'a jamais permis que de limiter le nombre des victimes des famines. En 1833, le système en vigueur repose sur l'oukase du 14 avril 1822 prévoyant la constitution de réserves intérieures et des subventions pour les années de disette. Il prescrit donc deux sortes d'interventions : la constitution de réserves de grains ou d'argent dans chaque gouvernement, par un prélèvement annuel soit en nature soit en argent auprès de toute « âme » (revizskaja duša, c'est-à-dire homme soumis à la capitation); des mesures exceptionnelles sous la forme de prêts d'État lorsque les réserves sont insuffisantes. En application de ces mesures, 40 gouvernements ont conservé le système des greniers ou magasins de réserve et 12 ont choisi de constituer des fonds monétaires. Ainsi, il existait 33311 de ces magasins en 1832 lorsque le pays eut à affronter les très mauvaises récoltes de 1833. Les fonds d'intervention ont aussi été utilisés par l'État pour porter des secours aux plus démunis ${ }^{16}$. Le système a donc fonctionné, surtout dans

15. A. Roslavskij, « Issledovanie o dviženii narodonaselenija v Rossii » (Étude du mouvement de la population en Russie », Vestnik imperatorskogo russkogo geografičeskogo obščestva, 1853 , p. $5,6,26$.

16. Pour un exposé du système et de son histoire, voir l'article "Prodovol'stvie narodnoe " (Approvisionnement national), in Brokgauz i Efron, Enciklopedičeskij slovar', t. 49, 1890, p. 354-361. 
les villages des paysans de la Couronne, mais il a également montré ses limites alors que toutes sortes de dysfonctionnements dont La Rüe nous donne un aperçu en réduisaient l'efficacité. En ce qui concerne les propriétaires et leurs serfs, Aleksandr Romanovič-Slavatinskij, s'appuyant sur des exemples des XVIII et $\mathrm{XIX}^{\mathrm{e}}$ siècles, explique que non seulement leurs obligations pour les années de disette manquaient de précisions, mais qu'en plus elles étaient loin d'être remplies ${ }^{17}$.

\section{Les colonies militaires}

Si rien ne prédisposait le baron de La Ruë à s'intéresser à la situation créée par les très mauvaises récoltes de 1833, il avait en revanche sous la main des documents sur les colonies militaires. Il prend l'initiative d'envoyer au maréchal Maison un rapport sur leur situation et pour cela se sert d'un article paru dans Le Spectateur militaire en $18288^{18}$.

Cet article, comme celui de 1830 qui le complète en donnant les grandes lignes du règlement de ces colonies ${ }^{19}$, n'est pas signé lors de sa parution, mais est attribué par les tables analytiques de la revue à un certain « commandant Chambaud ${ }^{20}$. Qui est cet auteur? Peut-être le baron de La Ruë lui-même. Nous n'avons pu l'établir avec certitude, mais La Ruë est l'un des quatre aides de camp qui accompagnent, en mai 1826, le duc de Raguse lors de son ambassade extraordinaire pour le couronnement de Nicolas I ${ }^{21}$. Pendant son séjour en Russie, le maréchal de Raguse s'est, selon ses propres dires, lié avec le comte de Witte ${ }^{22}$ et il est fort probable que c'est grâce à ce dernier que La Ruë a pu s'informer sur les colonies militaires.

Quel que soit cet auteur, il est certainement au courant des récentes publications françaises sur les colonies militaires. L'année 1825 a vu paraître, à Paris, deux traductions différentes de l'ouvrage de Robert Lyall sur les colonies militaires de

17. A. Romanovič-Slavatinskij, Dvorjanstvo v Rossii ot načala XVIII veka do otmeny krepostnogo prava (La noblesse en Russie du début du XVIII' siècle jusqu'à l'abolition du servage), Kiev, 1912, p. 303, 304, 337-339.

18. «Russie. Colonies militaires », Le Spectateur militaire, V, 1828, p. 242-253.

19. «Des colonies militaires de l'Empire de Russie», Le Spectateur militaire, IX, 1830, p. 467-506.

20. Le Spectateur militaire, Table générale des matières, Paris, 1880, p. 69. Parmi les officiers français dont nous avons pris connaissance de la carrière, aucun « Chambaud » n'est susceptible de s'être rendu en Russie peu avant 1828. En outre, il paraît invraisemblable qu'Ivan Pavlovič Chambaud (Šambo), secrétaire de l'impératrice, d'origine huguenote, ait écrit cet article.

21. AMAE, Personnel Série I, dossier 3388.

22. Marmont, Voyage du maréchal, duc de Raguse, en Hongrie, en Transylvanie, dans la Russie méridionale, en Crimée, et sur les bords de la mer d'Azoff, à Constantinople, dans quelques parties de l'Asie Mineure, en Syrie, en Palestine et en Égypte, I, Paris, 1837, p. 176. Sur le comte de Witte, voir infra note 29. 
Russie, publié à Londres l'année précédente ${ }^{23}$. Au cœur des préoccupations de ces auteurs et traducteurs, la puissance militaire de la Russie, son éventuel renforcement grâce aux colonies militaires, les menaces qui pèsent sur l'Europe, sur l'indépendance de ses États.

Rappelons quelques caractéristiques du système des colonies militaires, institution ancienne consistant en des unités de soldats paysans chargés de missions de défense aux frontières ou formant une armée de réserve et assurant leur propre subsistance grâce à la pratique de l'agriculture. Des colonies de soldats paysans ont existé dans les Empires romain et byzantin; de telles colonies assuraient encore au $\mathrm{XIX}^{\mathrm{e}}$ siècle la garde des frontières de la Suède et de l'Empire des Habsbourg. Il y en eut également en Ukraine au XVIII ${ }^{\mathrm{e}}$ siècle. Le projet lancé par Alexandre Ier en Russie est cependant nouveau par son ampleur, car le système est initialement appelé à remplacer le recrutement traditionnel et à s'étendre à terme à toute l'armée. Il poursuit deux buts principaux : réduire les dépenses liées à l'entretien d'une nombreuse armée et créer une nouvelle catégorie sociale (soslovie), jouissant de conditions matérielles (cadre et niveau de vie, instruction, conditions sanitaires) supérieures à celles des paysans et des soldats appelés à entrer dans ces colonies. La création de ces colonies relève bien d'un projet utopique de transformation de la société, cher à Alexandre Irer, même s'il se traduit par un enrôlement autoritaire de paysans, obligés de se soumettre aux règles militaires. Nous suivons sur ce point Richard Pipes ${ }^{24}$, tout en ne partageant pas l'ensemble de ses analyses. Sans entrer dans le détail, précisons un peu l'organisation initiale, du moins celle qui préside à la création des colonies à partir de 1816 . Chaque régiment colonisé est composé d'un bataillon de colons tenanciers (hozjaeva), de deux bataillons d'active et d'un de réserve. Les colons tenanciers sont choisis soit parmi les paysans des villages entrés dans la colonie, soit parmi les soldats du régiment colonisé. Il leur est attribué une terre, une maison où sont cantonnés deux soldats d'active qui les aident et qu'ils doivent nourrir. Les autres paysans des villages, appartenant le plus souvent à la Couronne et entrés dans les colonies, sont incorporés dans le régiment de réserve. Tous ont à la fois à mener des travaux agricoles et des exercices militaires. Leurs enfants, appelés cantonistes, sont divisés en trois classes d'âge. Les cantonistes aînés, de 12 à 18 ans, suivent les cours d'une école établie dans la colonie et sont formés aux exercices militaires. C'est parmi eux que sont recrutés les futurs soldats de réserve et d'active. Enfin, l'organisation prévoit également l'éducation des filles, leur mariage, le statut des invalides, les règles de succession ${ }^{25}$.

23. R. Lyall, Essai historique sur le système de la colonisation militaire en Russie, Paris, 1825, chez les Libraires du Palais Royal, dont le traducteur ne se fait pas connaître ; id., Notice sur l'organisation, l'administration et l'état présent des colonies militaires de Russie, Paris, Anselin et Pochard, Librairie pour l'art militaire, 1825, traduit par C. J. Ferry qui livre aussi ses «observations sur les résultats probables de l'établissement des colonies ».

24. R. E. Pipes, «The Russian military colonies, 1810-1831», Journal of Modern History, XXII, 3, 1950, p. 205-215.

25. Pour un aperçu général sur ces colonies, voir les articles de A. S. Lykošin, «Voennye poselenija », in Voennaja enciklopedija, t. VI, Saint-Pétersbourg, 1911, p. 608-611 et D. L. Schafly, "Military colonies », in The modern encyclopedia of Russian, Soviet and Eurasian 
La Ruë et ses contemporains ont une approche comparable des colonies militaires, dans laquelle se retrouvent un certain nombre de clichés plus ou moins fondés. Tous passent sous silence les colonies de Moguilev, les premières créées dès 1809. Tous opposent les colonies d'infanterie du Nord à celles de cavalerie du Sud et comparent leurs réussites respectives. Mais, leurs appréciations divergent. La Ruë et le maréchal de Raguse ${ }^{26}$ admirent la réussite de celles du Sud et soulignent l'échec de celles du Nord, opinion reprise comme évidente par l'historien du règne de Nicolas Ier, Paul Lacroix ${ }^{27}$. À l'inverse, Joseph Tanski, officier polonais réfugié en France, soutient que les colonies du Nord, fréquemment visitées par les souverains, ont un aspect plus flatteur que celles du Midi, moins soignées et moins prospères ${ }^{28}$. Ces auteurs, à des degrés divers, considèrent les premières comme le domaine d'Arakčeev, les secondes comme celui de Witte, oubliant que l'ensemble des colonies est placé jusqu'à la mort d'Alexandre Irr sous le haut commandement d'Arakčeev et obéit aux mêmes principes et règlements. Le comte de Witte ${ }^{29}$ a bien innové lors de l'établissement des colonies du gouvernement de Kherson et ses nouveautés sont appréciées d'Arakčeev qui souhaite les faire adopter à l'ensemble des colonies. L'opposition entre deux systèmes qui seraient différents au Nord et au Sud, telle que la présentent nos auteurs, est sans doute liée à l'époque de leurs investigations sur les colonies militaires, époque postérieure à la disgrâce d'Arakčeev (1826) où s'exprime toute la rancœur accumulée contre le favori d'Alexandre Ier, et à leurs informateurs, dont le comte de Witte lui-même. Cependant, si colonies du Nord et du Sud appartiennent à l'origine à un système commun, elles diffèrent profondément par les milieux humains dont sont issus leurs membres, celles de Novgorod étant implantées parmi des populations grand-

history, t. 22, Gulf Breeze, FL, 1981, p. 80-84. Pour un exposé détaillé de l'organisation initiale et de la réforme de 1826, voir «Des colonies militaires de l'Empire de Russie », art. cit., p. 467-506.

26. Marmont a visité les colonies du Sud en 1835. Il en donne une description détaillée, op. cit., p. $195-262$.

27. Paul Lacroix, Histoire de la vie et du règne de Nicolas I ${ }^{e r}$, empereur de Russie, Paris, 18641868, 5 vols., ici vol. IV, 1867, p. 67.

28. Joseph Tanski, Tableau statistique, politique et moral du système militaire de la Russie, Paris, 1833, p. 136-138.

29. Ivan Osipovič Witte, officier de cavalerie (1781-1840) : son père était d'origine polonaise et sa mère grecque. Il s'est engagé en 1809 comme volontaire dans l'armée de Napoléon dont il fut un agent secret dans le grand-duché de Varsovie. Il revint au service de la Russie en 1812 et prit part aux campagnes contre Napoléon. En 1817, il fut chargé de la formation d'une division de uhlans du Boug et de son établissement dans la colonie militaire de Tchougouev. À partir de l'année suivante, il s'occupa de l'installation de colonies militaires de cavalerie dans le Sud et se distingua en obtenant l'approvisionnement de la division par les colons au bout des trois ans qu'il avait fixés. Après de brillants succès militaires dans la campagne contre la Turquie (1829), qui lui valent d'être promu général, et en Pologne (1831), il devint l'année suivante inspecteur des colonies de cavalerie. Le témoignage de A. Ja. Storoženko, major chargé des travaux d'arpentage dans les colonies du Boug puis nommé à l'état-major de Witte qui commandait alors les colonies des gouvernements de Kherson et d'Ekaterinoslav, montre le contrôle étroit qu'y exerçait Arakčeev (N. I. Ušakov, « Vospominanija ob Andree Jakovleviče Storoženke » (Souvenirs sur Andrej Jakovlevič Storoženko), Russkij arhiv,1873, p. 1722-1735. 
russiennes, comptant de nombreux vieux-croyants, attachées à leurs traditions et réfractaires aux innovations et à la discipline militaire, celles du Sud étant formées sur des terres nouvellement peuplées et mises en valeur, où les cosaques ou d'autres communautés paysannes militarisées aux origines ethniques et aux statuts divers formaient une grande partie du peuplement avant leur incorporation dans les colonies militaires ${ }^{30}$. Par ailleurs, les réformes de 1831-1832, adoptées au lendemain de l'insurrection des colonies de Novgorod et sur lesquelles nous aurons à revenir, donnent des noms différents aux établissements d'infanterie et de cavalerie et introduisent des différences notables dans leur organisation.

Les appréciations sur la façon dont les colonies ont atteint leurs objectifs économique (réduction des dépenses), agricole ou militaire, varient d'un auteur à l'autre. L'enthousiasme du maréchal de Raguse, que rien ne vient tempérer lors de sa tournée des colonies du Midi, n'est partagé ni par Joseph Tanski ni par le baron de La Ruë. Pour Tanski, très sceptique sur l'intérêt financier des colonies, d'indéniables progrès agricoles ont été obtenus grâce aux grands défrichements de la région de Novgorod, mais au prix d'une détérioration grave de la condition des paysans de la Couronne incorporés dans les colonies. Enfin, il voit dans les colons armés un risque plus grand pour le despotisme russe que pour les ennemis du dehors ${ }^{31}$. Le baron de La Ruë a, dans l'ensemble, des positions beaucoup moins tranchées. Elles portent surtout sur les colonies de cavalerie, car il considère que celles d'infanterie ont été supprimées lorsqu'elles ont été transformées en districts de soldats laboureurs (novembre 1831). Il pense que les objectifs initiaux ont été en grande partie atteints dans les colonies de cavalerie mais il insiste sur le malheur des colons, malgré leur prospérité, et le mécontentement des officiers. Il rejoint Tanski sur la menace pour la tranquillité de l'État, mais voit dans les colonies du Midi une place d'armes contre la Turquie et l'Autriche. Les colonies militaires ont continué à susciter des appréciations divergentes. Parmi les officiers qui y ont travaillé et ont écrit leurs souvenirs après leur suppression, en 1856 dans le Nord, en 1866 pour les colonies de cavalerie du Sud, la plupart décrivent un travail harassant, une discipline de fer et des résultats en complet décalage avec ceux qui avaient été escomptés ${ }^{32}$. Pavel P. Karcov prend systématiquement le contre-pied des critiques émises dans ces souvenirs et défend la politique d'Arakčeev, opposant à tous les

30. E. I. Družinina, Južnaja Ukraina v 1800-1825 gg. (L'Ukraine méridionale, 1800-1825), Moscou, 1970, p. 70-73; id., Južnaja Ukraina v period krizisa feodalizma, 1825-1860 gg. (L’Ukraine méridionale pendant la crise du féodalisme, 1825-1860), Moscou 1981, p. 12-15.

31. J. Tanski, op. cit., p. 140-152.

32. Parmi ces témoignages, celui de E. F. von Bradke est particulièrement intéressant, car il porte tant sur les colonies de Novgorod que sur celles du Midi. Il montre l'ampleur des travaux entrepris, l'énergie qu'il a déployée dans des missions pour lesquelles il n'était pas formé et juge qu'aucun des buts poursuivis dans les colonies militaires n'a été atteint, particulièrement dans celles du Nord. Pour lui, cette institution dans toute sa complexité pouvait sembler brillante, vue de l'extérieur et superficiellement, alors qu'en son sein régnaient le malheur et l'abattement («Avtobiografičeskie zapiski Egora Fedoroviča Fon-Bradke » (Notes autobiographiques d'Egor Fedorovič von Bradke), Russkij arhiv, 1875, t. I, p. 13-53 ; t. III, p. $257-$ 294). 
dysfonctionnements décrits les règlements des colonies militaires ${ }^{33}$. C'est sans doute le grand décalage entre ces règlements et leur application - sur lequel insiste l'étude bien documentée de Andrej N. Petrov ${ }^{34}$ - qui est à l'origine des divergences d'appréciations, les défenseurs s'appuyant sur les textes, les détracteurs du système sur les situations créées au nom de ces mêmes règlements.

Mais, dans les années 1830, admirateurs et sceptiques se retrouvent sur un thème : ces colonies représentent le passage de l'état sauvage à la civilisation. Lyall considère qu'un des principaux objectifs poursuivis par Alexandre Ier est de hâter le progrès de la civilisation dans son empire et que l'institution des colonies constitue le moyen le plus efficace pour atteindre ce but ${ }^{35}$. Tanski voit aussi dans ce système le seul moyen d'amener à la civilisation ce pays plongé dans la barbarie ${ }^{36}$. Le maréchal de Raguse admire l'ordre, l'économie et le degré très avancé en civilisation d'une population qui, vingt ans auparavant, était barbare, presque sauvage ${ }^{37}$. Tous ont été impressionnés par leur visite de Voznessensk, le chef-lieu des colonies militaires de Nouvelle Russie, systématiquement montré aux rares Occidentaux de passage, et ont admiré l'agencement symétrique des maisons, les superbes bâtiments de service public, les écoles, les hôpitaux, le bon entretien des routes ... De la même façon, le comte Kočubej, chancelier des Affaires intérieures, écrit à Arakčeev à propos des colonies de Novgorod qu'en allant de celles construites sur le Volkhov sur la rive d'en face, il est passé d'une région civilisée à une contrée barbare ${ }^{38}$. Prosper de Barante, ambassadeur de France en Russie, a, lui aussi, visité Voznessensk et il note que « ces résultats, au premier coup d'œil paraissent très beaux. En y réfléchissant, on voit qu'ils sont très beaux seulement pour la Russie ». Et, persuadé que les terres seraient demeurées incultes si elles n'avaient été allouées aux colons, il conclut : « la superbe colonie de Voznessensk est un signe évident de l'état reculé où se trouve encore la Russie, et peut-être de tels établissements ne sont-ils pas conçus dans un esprit qui puisse hâter ses progrès. $»^{39}$ En fait, pour tous - et sur ce point Barante ne fait pas exception - , les paysans de la Couronne, leurs villages et leurs terroirs, qui constituent la majorité des habitants enrôlés et

33. P. P. Karcov, «O voennyh poselenijah pri grafe Arakčeeve» (Les colonies militaires au temps d'Arakčeev), Russkij vestnik, 1890, t. 206, p. 139-171 ; t. 207, p. 82-113 : t. 208, p. 75121.

34. Le colonel Andrej Nikolaevič Petrov, qui a fait partie de l'encadrement militaire des colonies, a écrit une étude historique sur leur établissement et leur administration de 1809 à 1826 , publiée dans le recueil des éditions de Russkaja Starina : Graf Arakčeev i voennye poselenija. 1809-1831 (Le comte Arakčeev et les colonies militaires, 1809-1831), Saint-Pétersbourg, 1871, p. 87-207 (cité infra Petrov). Il y oppose souvent discours et faits, mesurant le miracle des marais transformés en champs florissants à l'aune du nombre de personnes devenues des ombres errantes et concluant qu'il s'agissait en fait d'une terre de sang et de larmes (p. 206207).

35. R. Lyall, Essai historique sur le système de la colonisation, op. cit., p. 43.

36. J. Tanski, op. cit., p. 152.

37. Marmont, op. cit., p. 221-222.

38. Lettre citée par P. P. Karcov, art. cit., t. 207, p. 87.

39. Prosper de Barante, Notes sur la Russie, 1835-1840, Paris, 1875, p. 162-163. 
des terres réquisitionnées par les colonies, formaient un monde inculte, sauvage et barbare. Nous retrouvons là l'ignorance ou le mépris de la Russie traditionnelle que nous avons pu entrevoir dans le rapport sur la famine et les mesures de prévention des disettes. Cette ignorance ou ce mépris prennent une forme paradoxale dans le cliché d'une région de Novgorod peu habitée, inculte, voire déserte, repris par La Ruë, Raguse et Tanski ${ }^{40}$. La région de Novgorod a, en 1811, une densité de population un peu supérieure à celle du gouvernement de Kherson, mais de l'ordre du tiers de celle de Kharkov. Certes, elle ne connaît pas l'essor démographique rapide de la Nouvelle Russie en ce premier tiers du XIX ${ }^{\mathrm{e}}$ siècle $^{41}$. Mais il n'y a pas de raison objective de la présenter comme un désert vide d'hommes au moment de la création des colonies militaires et de l'opposer si catégoriquement en cela aux régions méridionales.

Enfin, cette perception globale de l'état arriéré de la Russie, du mauvais fonctionnement de son administration, de sa corruption donne un relief particulier aux personnages brillants, appartenant à l'élite occidentalisée qui accueillent nos visiteurs de marque. L'enthousiasme du maréchal de Raguse est entretenu par la magnifique réception que lui réservent le comte Voroncov à Odessa, le comte de Witte et les officiers de cavalerie sous ses ordres dans les colonies. Le baron de La Ruë, également admiratif des actions entreprises par ces éminents personnages, a certes des appréciations plus pondérées mais il n'échappe pas à leur ascendant et surévalue leur rôle et leur indépendance face au pouvoir central.

En fait, à l'époque de la mission de La Ruë dans le Sud de la Russie, les colonies militaires ont été profondément réformées par Nicolas Ier. Ce dernier donne au système son expansion maximale, mais il le vide de sa dimension utopique en rapprochant les statuts des colons de ceux des paysans de la Couronne, en abandonnant le projet de faire des colonies le mode unique de recrutement de l'armée et en supprimant leur commandement militaire séparé. Ces réformes, commencées dès $1826^{42}$, poursuivies en 1829 en séparant les commandements des régiments d'active, de réserve et de colons ${ }^{43}$, prennent un tour radical en 1831-1832. Les colonies d'infanterie de Novgorod sont alors transformées en districts de soldats

40. Marmont, op. cit., p. 496 ; J. Tanski, op. cit., p. 119.

41. Pour la population des gouvernements de la Russie d'Europe, voir A. G. Rašin, Naselenie Rossii za 100 let (1811-1913) (L'évolution de la population de la Russie en 100 ans, 18111913), Moscou 1956, p. 28-29. Ainsi, le gouvernement de Novgorod a une population de 765800 h. en 1811, 825400 h. en 1838 ; celui de Kherson : 370400 h. en 1811, 765800 en 1838, celui de Kharkov de 1030000 en 1811, 1334000 en 1838. En rapportant ces chiffres aux superficies de ces gouvernements, nous obtenons des densités par verste carrée de 7,35 puis 7,90 pour Novgorod, 5,95 puis 12,30 pour Kherson, 21,50 puis 27,80 pour Kharkov.

42. Dès octobre 1826, le commandement séparé et unifié des colonies militaires est remplacé par des commandements de chaque région (Novgorod, Moguilev, Kherson-Ekaterinoslav, Ukraine des faubourgs (Slobiska Ukrajna)) et leur administration rattachée à l'État-Major de l'empereur (Alan D. Ferguson, «The Russian military settlements, 1825-1866 », in A. D. Ferguson, A. Levin, eds., Essays in Russian history, Hamden, CT, 1964, p. 110). De plus, le règlement du 19 novembre/1 ${ }^{\text {er }}$ décembre 1826 prévoit la dispense des maîtres colons des exercices militaires (P. Lacroix, op. cit., t. II, p. 381-382). 
paysans qui conservent les terres des anciens régiments colonisés. Les maîtrescolons (hozjaeva) se voient attribuer quinze déciatines et sont collectivement tenus de nourrir les troupes cantonnées dans leur district. Des aides civils les assistent dans leurs travaux agricoles. Devant s'acquitter annuellement de l'obrok et soumis aux règles de recrutement des autres sujets de l'empire ${ }^{44}$, ils ont désormais un statut semblable à celui des paysans de la Couronne. Dans les colonies militaires de cavalerie, qui conservent leur appellation, il y a aussi séparation, du moins théoriquement, des travaux agricoles et des exercices militaires. Les maîtres-colons sont tenus à des prestations en nature (trois jours de travail hebdomadaire pour la colonie) et soumis au recrutement comme les cosaques de Petite Russie ${ }^{45}$. À partir de 1832, l'administration des districts de soldats laboureurs et des colonies militaires est confiée à un département du ministère des Armées, lequel a également sous sa responsabilité, à partir de 1835 , les autres troupes irrégulières ${ }^{46}$. Cette organisation apparaît clairement dans la présentation du ministère des Armées faite à l'occasion des vingt ans de règne de Nicolas Ier. En annexe du compte rendu sur l'administration de l'armée de terre, viennent les colonies militaires fortes de 374478 âmes des deux sexes en 1826, de 713311 en 1850, et les régiments irréguliers (cosaques et divers escadrons) forts de 1116726 âmes des deux sexes en 1826 et 2086546 en $1850^{47}$.

Sur le terrain, le baron de La Rüe voit la situation des colonies de l'Ukraine des faubourgs et de la Nouvelle Russie dont chacune a un vécu différent. Elles lui apparaissent comme des îlots de prospérité dans des régions ravagées par la famine. Ses rapports, par leur approche des différentes facettes de réalités complexes, apportent bien des informations que n'aurait pas comportées une enquête à visée purement stratégique, comme l'avait définie le maréchal Maison en envoyant son aide de camp en mission. Ils s'avèrent intéressants également par les interprétations erronées qu'ils contiennent, car celles-ci sont révélatrices de conceptions largement répandues à cette époque.

edith.ybert@cegetel.net

44. Ibid., p. 116-118.

45. Ibid., p. 119.

46. A. S. Lykošin, art. cit., p. 611.

47. I. F. Dubrovin, «Materialy i čerty k biografii imperatora Nikolaja I i k istorii ego carstvovanija » (Matériaux et grandes lignes d'une biographie de l'empereur Nicolas Ire et d'une histoire de son règne), Sbornik imperatorskogo russkogo istoričeskogo obščestva, SaintPétersbourg, 1896, p. 420-425. 


\section{I Rapport sur l'état agricole des gouvernements du midi}

Monsieur le Maréchal,

Pour remplir les instructions qui m'ont été remises par votre Excellence, je me suis attardé à réunir le plus de documents possibles sur l'état agricole des gouvernements qui souffrent en ce moment de la disette. J'ai parcouru la plupart de ces provinces et partout en constatant l'état de souffrance de ces populations, j'ai recherché les causes qui ont concouru à augmenter le mal, et les efforts qui ont été faits pour le diminuer, ainsi que les conséquences prolongées, qui seront la suite forcée de cet état de choses.

Les gouvernements où la famine se fait sentir, sont ceux de : Géorgie, Caucase, Astracan, Cosaques du Don, Saratoff, Tamboff, Woronège, Koursk, Slobodes d'Ukraine, Jekatérinoslaw, Tauride, Kerson, Bessarabie, Poltava, Mohilew, Smolensk et Witepsk. La récolte avait été à peu près nulle dans tous ces gouvernements, surtout dans ceux de l'Est et le plus au midi. Les gouvernements de Poltava, Mohilew, Smolensk et Witepsk, que j'ai aussi parcourus, ont moins souffert ${ }^{48}$. La récolte avait manqué dans quelques districts seulement, et les semailles pour cette année ont pu être faites complètement dans ces quatre gouvernements. Dans la plupart des autres gouvernements, la chaleur et l'extrême sécheresse avaient par leur durée inaccoutumée fait également manquer les foins, de là vient que ces contrées ont perdu la plus grande partie de leurs bestiaux, par suite de la mortalité ou par l'émigration dans les provinces plus à l'ouest. Cette perte sera très difficilement réparée ; les propriétaires et les paysans sont dans l'impossibilité de rien faire à cet égard. Le mauvais état des affaires des premiers, et la misère habituelle des seconds ne leur permettent en ce moment que de subvenir à des besoins plus pressants. Nulle part le gouvernement, ni l'autorité locale, n'ont pris des mesures pour les aider sous ce rapport, excepté dans les gouvernements de Tauride et de Kerson, où les réserves en bestiaux des colonies de cavalerie ${ }^{49}$ ont pu être distribuées à ceux qui avaient le plus souffert.

En tous temps, en Russie, une épizootie cause des dommages que plusieurs années ne suffisent pas pour réparer, malgré l'étendue des pâturages qui semble favorable à la multiplication des bestiaux. Les contrées où le foin a manqué sont donc aujourd'hui dans le cas de celles qui auraient été ravagées par une épizootie

48. La liste donnée correspond bien à celle des gouvernements où le recensement et le recrutement décrétés en 1833 ont été suspendus en raison de la famine, et comporte en plus les gouvernements de Saratov, Koursk et Tambov, ce dernier étant touché par la disette de 1834, comme nous l'avons vu précédemment. En revanche, les trois gouvernements de Mohilev, Smolensk et Witepsk ne font pas partie de la liste des gouvernements exempts de recensement et de recrutement, à la différence de celui de Poltava, liste donnée par V. M. Kabuzan dans Narody Rossii v pervoj polovine XIX veka (Les peuples de Russie dans la première moitié du XIX siècle), Moscou, Nauka, 1992, p. 65. Cette liste est donc beaucoup plus étendue que celle donnée par Slovcov («Istoričeskoe i statističeskoe obozrenie neuroažev... », art. cit., p. 471-472) et ne comporte pas Tchernigov.

49. Elles font partie des colonies militaires auxquelles est consacré le second rapport publié ciaprès. 
meurtrière. Dans toutes les directions que j'ai parcourues, j'ai trouvé les routes semées de cadavres de chevaux et de bestiaux, comme celles que suivrait une armée en déroute ${ }^{50}$.

La disette avait été pressentie dans le milieu de l'été dernier. Le printemps avait annoncé peu de chose pour l'année, et la longue sécheresse de l'été vint ensuite enlever tout espoir de récolte. Chacun avait aperçu la calamité qui menaçait ces contrées. Soit imprévoyance, soit impossibilité pécuniaire de le faire, il n'y eut d'abord que quelques particuliers qui s'occupèrent d'approvisionnement pour eux et leurs paysans ${ }^{51}$. Tout se borna alors de la part du plus grand nombre à tâcher de se procurer ce qu'il fallait pour les semailles suivantes et ce résultat n'a pu être atteint complètement dans les gouvernements qui ont le plus souffert ${ }^{52}$. J'ai, en questionnant un grand nombre de paysans, constaté que les semailles n'avaient été faites, terme moyen, qu'à peine aux deux tiers de terres ordinaires, soit par le défaut de grains, soit par suite des émigrations.

Peu d'administrateurs avaient songé à prévenir la famine, par de sages mesures. On dit même que dans plusieurs gouvernements l'on avait négligé d'empêcher la sortie double de l'année précédente, quoique l'importation y dût être considérée comme bien difficile pour plus tard ${ }^{53}$. La seule attention des gouverneurs fut de paraître sans inquiétude vis-à-vis de leurs administrés et de tenir à ce que le silence

50. T. de Lagrené, chargé d'affaires à l'ambassade de France à Saint-Pétersbourg signale dans plusieurs localités l'interruption du service des postes par « manque absolu de chevaux qui sont morts, ou dont il a fallu se défaire parce qu'il devenait impossible de les nourrir » (AMAE, Correspondance politique Russie, $n^{\circ}$ 187, f. 183). Il mentionne aussi que, dans la Russie méridionale, les 9/10 des troupeaux ont été tués et salés par les propriétaires. Le reste a été déplacé dans des gouvernements moins maltraités par la disette (ibid., f. 272). Paul Lacroix signale que dans les campagnes, en Ukraine et en Crimée, dans les provinces les plus fertiles et renommées pour leurs pâturages, « on tuait le bétail, faute de pouvoir le nourrir, les propriétaires de troupeaux abandonnaient ces troupeaux à quiconque s'engageait à leur en rendre la moitié au printemps prochain. », P. Lacroix, Histoire de la vie et du règne de Nicolas ${ }^{e r}$, op. cit., vol. VI, 1869 , p. 341.

51. Nous avons vu que ce jugement n'est pas corroboré par les faits. Quelle qu'ait été l'insuffisance des moyens mis en œuvre, ces derniers ne relèvent pas des seuls particuliers. La suite du rapport le dément aussi en partie, puisque les actions des pouvoirs publics y sont évoquées.

52. T. de Lagrené rapporte que «malgré les envois d'argent et de grains, qui absorbent les ressources déjà restreintes du Trésor, il ne parvient pas même à soulager provisoirement la misère des populations affamées. Le peu de blé que la difficulté des transports a permis d'envoyer sur les points ne laissant pas même les moyens d'ensemencer les terres, a été pillé par les habitants insurgés et n'a servi qu'au soulagement momentané de leur détresse. » (AMAE, Correspondance politique Russie, $\mathrm{n}^{\circ}$ 187, f. 18).

53. Il y a bien eu des grains exportés de Russie au début de l'année 1833. L'aperçu du commerce extérieur de la Russie montre que 1,5 million de četvert de grains ont été exportés pour 200000 importés (Journal de Saint-Pétersbourg, 21 juillet / 2 août 1834). Ces exportations ont très peu emprunté les ports de la mer Noire ou de la mer d'Azov, débouchés naturels des zones méridionales. Les données sur ces ports montrent des exportations en chute par rapport à l'année précédente de plus de $60 \%$ pour le froment, de près de $90 \%$ pour le seigle, près de $80 \%$ pour l'orge et presque nulles pour l'avoine, et encore beaucoup plus faibles en 1834 (E. I. Družinina, Južnaja Ukraina v period krizisa feodalizma. 1825-1860 gg. (L'Ukraine méridionale à l'époque de la crise du féodalisme. 1825-1860), Moscou, Nauka, 1981, p. 186). En 1833, le blé a figuré pour la première fois dans la liste des marchandises importées par Odessa (Journal de Saint-Pétersbourg, 3/15 mars 1834). 
fût gardé sur l'état des choses dans les lieux qui devaient souffrir de la famine ouverte. On a pu par là retarder le moment où la disette a commencé à se faire sentir et contribuer à retarder aussi l'augmentation dans le prix du blé. Ce moyen a épargné de grandes inquiétudes, et il a été assez constamment suivi, pour qu'aujourd'hui encore, les habitants d'un gouvernement ignorent à peu près où en sont les choses dans le gouvernement voisin.

Ce ne fut que vers la mi-août 1833 que le gouvernement publia officiellement les premières mesures générales, bien qu'il dût être instruit de l'état des récoltes par les rapports mensuels des gouverneurs civils.

Cependant on cite des gouverneurs qui n'avaient point attendu les ordres de l'autorité suprême pour arrêter une partie des effets de la calamité menaçante. Dès la fin juillet, le comte Woronzoff ${ }^{54}$ avait fait d'immenses achats de blé qui devait être plus tard distribué à un prix modéré dans les gouvernements du midi soumis à sa juridiction. Soit que le comte Woronzoff fut plus habile ou plus indépendant dans les actes de sa gestion, il ne crut pas devoir attendre pour prévenir le mal, qu'il fut devenu trop grand. Sa position favorable à Odessa et sa grande fortune personnelle aidèrent aux mesures qu'il crut devoir prendre à temps. On évalue à trois millions de roubles le chiffre des acquisitions en blé faites par ses soins. Il se servit pour cette opération de la maison Stieglitz de Saint-Pétersbourg, qui a un comptoir à Odessa et qui préparait déjà depuis quelque temps une très grande spéculation commerciale sur les divers produits de la Russie méridionale ${ }^{55}$. Cette maison avait

54. Mihail Semenovič Voroncov (1782-1856), gouverneur général et vice-roi de la Nouvelle Russie et de la Bessarabie à partir de 1823, administrateur ayant acquis une excellente éducation à l'anglaise, connu pour son action efficace de mise en valeur de la région et pour son libéralisme éclairé. Il reçut aussi la délicate mission de gouverner le Caucase à partir de 1844 .

55. Le secrétaire du prince Voroncov, M. P. Ščerbinin, rend compte de l'action de son maître pour lutter contre la famine, action beaucoup moins personnelle que ne le pense La Ruë. Il explique que l'aide a été envoyée par le «gouvernement plein de sollicitude (popečitel'noe pravitel'stvo) », tandis que Voroncov a contrôlé les travaux des divers comités chargés d'examiner les besoins, de distribuer les subsides en argent et en nature, de fournir les grains pour les prochaines semences et le bétail pour les travaux des champs, tout en suivant avec impartialité les diverses opérations. Il s'est en effet adressé à une maison commerciale grâce à laquelle ont pu être évités les dysfonctionnements dans l'acheminement et la distribution des denrées. Voroncov a lui-même inspecté tous les territoires sous sa juridiction et est parvenu à limiter les dégâts et les dépenses (M. P. Ščerbinin, Biografija generala fel'dmaršala knjazja Mihaila Semenoviča Voroncova (Biographie du général feld-maréchal, prince Mihail Semenovič Voroncov), Saint-Pétersbourg, 1858, p. 202-203). L'auteur, sans doute au courant des reproches adressés par l'empereur à Voroncov à propos de cette famine, a rédigé son texte avec circonspection. En effet, Nicolas I ${ }^{\text {er }}$ a estimé que le vice-roi avait manqué à ses devoirs et lui a envoyé en octobre 1833 une lettre de reproche. En fait, Voroncov avait envoyé plusieurs rapports au Conseil des ministres mais pas à l'empereur lui-même (A. L. H. Rhinelander, Prince Michael Vorontsov, viceroy to the tsar, Montreal, McGill-Queen's University Press, 1990, p. 103-104). Le différend portait sur le manque d'information et non sur l'action ellemême, mais il est fort probable que le récit de La Ruë est inspiré par de zélés défenseurs de Voroncov. Il nous est impossible de préciser en quoi la grande fortune de Voroncov aida aux mesures qu'il prit. C'est vraisemblablement en tant que propriétaire de vastes domaines en Nouvelle Russie qu'il eut aussi à intervenir personnellement. De fait, les visiteurs occidentaux de passage à Odessa, tant le maréchal Marmont, comte de Raguse, que Prosper de Barante, ambassadeur de France en Russie de 1835 à 1841, vantent son rôle de bienfaiteur, subvenant à des dépenses publiques lorsque l'allocation officielle est insuffisante. Les acquisitions de blé pour trois millions de roubles dont il est question doivent cependant avoir été faites avec des 
au moyen de nombreux agents établi des relations dans toutes les provinces. Les mêmes agents furent aussitôt chargés d'assurer l'opération que le comte Woronzoff confia aux soins de la maison Stieglitz ${ }^{56}$. Ils réunirent en effet une assez grande quantité de blé, mais cette active prévoyance du comte au profit des gouvernements soumis à son administration, contribua à augmenter la détresse des gouvernements voisins, qui s'en plaignirent même, d'abord comme d'une spéculation de commerce tentée par la maison Stieglitz.

Comme il a été dit, les premières mesures générales furent officiellement publiées vers la mi-août. Alors seulement on songea dans les gouvernements mal traités à retenir le peu de blé qu'il y avait. La permission d'importation sans aucune espèce de droits fut accordée ; injonction fut faite aux propriétaires de s'occuper de la subsistance de leurs paysans, et l'importation fut facilitée par tous les moyens possibles dans les lieux qui souffraient le plus.

Néanmoins l'importation a été et sera peu considérable, les distances énormes, les difficultés de transport accrues par la mort des bestiaux et la direction en sens opposé des cours des rivières, mettent le blé à un taux bien au-dessus des ressources des consommateurs ${ }^{57}$. Il y a souffrance réelle de la part du paysan qui cherche à tromper sa faim par une nourriture qui ne fera que compromettre sa santé et amener de nombreuses et graves maladies parmi ces malheureux ${ }^{58}$. On peut prévoir que lors même la récolte de cette année serait favorable, les souffrances prolongées des paysans de ces contrées détermineront une mortalité qui ne sera pas moindre les années prochaines que celle-ci ${ }^{59}$. Et au dire même des administrateurs, le chiffre des mortalités s'est fort élevé cette année, sans qu'ils sachent du reste le préciser,

avances du gouvernement. L'action efficace de Voroncov se déploya dans le cadre des institutions de l'Empire. Par exemple, 10000 četvert de farine furent prélevés des réserves de l'armée pour l'alimentation des riverains de la mer Noire (I. F. Dubrovin, « Materijaly i čerti k biografii Nikolaja I... », art. cit., p. 353).

56. Cette «maison Stieglitz» est l'une des nombreuses entreprises lancées par Ljudvig Ivanovič Stiglic (1788-1843), banquier d'origine allemande, possédant de florissantes affaires commerciales à Saint-Pétersbourg et à Narva, des usines de tous genres et de vastes élevages de moutons mérinos en Ukraine méridionale. Il reçut le titre héréditaire de baron en 1826.

57. Si les conséquences de la famine (mort des troupeaux et des chevaux de trait) viennent bien s'ajouter aux caractéristiques permanentes du pays (grandes distances et faible développement des moyens de communication), il faut cependant relativiser le propos sur deux points : la direction contraire des fleuves ralentit le transport fluvial des marchandises, mais ne l'empêche pas, un important système de halage ou de portage étant encore en place avant la diffusion de la machine à vapeur (A. V. Dulov, op. cit., p. 126-127) ; l'importation du blé dépend surtout des ressources que les autorités de tutelle des paysans y consacrent et non des consommateurs euxmêmes.

58. La consommation de toutes sortes de substances entrant dans la composition du pain est attestée par Slovcov qui évoque le mélange de farine avec des vannures et des tourteaux ainsi que des préparations à base d'herbes bouillies (art. cit.,p. 486), et par T. de Lagrené qui signale la fabrication de pain composé d'un tiers de farine et de deux tiers d'écorce de bouleau et de paille hachée, ainsi que la consommation de glands et du résidu des bleds servant à produire l'alcool (AMAE, Correspondance politique Russie, $n^{\circ}$ 187, f. 271-272).

59. Nous avons vu qu'en fait 1833 constitue bien un pic de mortalité. La mortalité décroît fortement en 1834 pour remonter en 1835, année de mauvaise récolte (A. Roslavskij, art. cit., p. 5$6)$. 
parce que excepté dans les villes, ils n'ont pas de moyens réguliers de constater le mouvement annuel de la population, laissant ces soins de détail aux seigneurs propriétaires des paysans, plus directement intéressés ${ }^{60}$. Le gouvernement s'aperçut donc bientôt, que l'importation se réduirait à peu de choses, à cause des distances et de la pauvreté des consommateurs, qui ne pourraient acheter ce blé au prix où il devait s'élever rendu chez eux. Alors on s'est attaché à un autre moyen de rendre la disette moins forte. Le gouvernement a fait délivrer des passeports gratuits aux gens qui iraient chercher de l'ouvrage ou leur subsistance dans les provinces où la moisson n'avait pas manqué61. Mais cette mesure qui a pu s'appliquer avec quelques succès dans les gouvernements de Tamboff, Saratoff, Koursk et Kharkoff, est devenue un embarras extrême pour les autorités dans d'autres contrées. Par exemple, les habitants de certaines parties du Caucase, où la récolte a entièrement manqué, ont déserté en masse leur pays. C'étaient des colons établis au Caucase par les soins de la tzarine. Les familles avaient été conduites de force des domaines de la couronne, où la population était surabondante, dans ces contrées désertes. Leur séjour y était contraire à leurs goûts et à leurs anciennes habitudes. Cette répugnance s'est perpétuée et lorsque la famine s'est fait sentir, ils ont saccagé, détruit leurs habitations et se sont mis en route avec leurs bestiaux pour rentrer en Russie. Ils sont arrivés au nombre de 30000 dans les gouvernements de Koursk et de Kharkoff, semant leur longue route de leurs morts et marquant leur passage par des actes de violence et de graves désordres. Les autorités de ces deux gouvernements reçurent l'ordre de les empêcher de continuer leur marche ${ }^{62}$. Les émigrants sont

60. Si, avant le début des années 1830 , seule la population mâle et soumise à la capitation était recensée lors des « révisions », à partir de ces années-là, le ministère des Affaires intérieures fait dénombrer, dans les gouvernements, par l'administration ou par la police, les membres des catégories non soumises à la capitation (V. M. Kabuzan, op. cit., p. 91-92). Par ailleurs, la mortalité de la population orthodoxe, enregistrée par l'Église, ne tient compte ni des suicidés ni de ceux qui n'ont pas été enterrés religieusement, du fait de l'accumulation de cadavres lors des épidémies ou autre calamités (A. G. Rašin, Naselenie Rossii..., op. cit., p. 41). Il y a donc à l'époque de la mission de La Ruë des données démographiques fiables, mais sujettes à plus d'erreurs par sous-estimation des morts une année de famine. Celles de 1833 ne sont pas encore connues des administrateurs quand La Ruë effectue son voyage en avril-mai 1834.

61. La délivrance gratuite de passeport pour que les paysans puissent gagner une région épargnée par la disette fait partie des mesures utilisées régulièrement en cas de mauvaise récolte. Elle n'est pas une alternative à l'importation de céréales, mais se pratique parallèlement à d'autres mesures (Slovcov, art. cit., p. 486). Paul Lacroix, à l'opposé de La Ruë, fait état d'immenses achats de blé venant d'Asie Mineure, d'Égypte et d'Autriche (op. cit., vol. VI, p. 419).

62. Par Caucase, il faut entendre le gouvernement de Stavropol qui portait alors ce nom et formait avec la région voisine, celle des cosaques de la mer Noire, le Caucase du Nord. La colonisation russe s'y développe à partir de la fin du règne de Catherine II. L'arrivée de colons venus des régions centrales s'y échelonne ainsi : de 1796 à 1815, dans le seul gouvernement du Caucase, 56000 colons venant majoritairement du gouvernement de Koursk ; de 1796 à 1811 , dans l'ensemble du Caucase du Nord, 100000 colons; de 1816 à 1834, 90000 colons dans le gouvernement du Caucase, 50000 sur la terre des cosaques de la mer Noire. La mortalité était très forte parmi ces arrivants qui avaient du mal à s'acclimater à leurs nouvelles conditions de vie et ne recevaient pas de soutien suffisant des autorités (V. M. Kabuzan, Emigracija i reemigracija v Rossii $v$ XVIII-načale $X X$ veka, (Émigration et réémigration en Russie, du XVIII ${ }^{e}$ au début du XXe siècle), Moscou, 1998, p. 55). C'est donc principalement du gouvernement de Stavropol qu'arrivent les 30000 réfugiés dont parle La Ruë, chiffre très important mais 
donc aujourd'hui un surcroît de charge pour ces deux gouvernements et un embarras de plus pour les autorités, dont toute la surveillance ne parvient pas à empêcher qu'ils ne commettent, poussés par la misère et le désespoir, les actes de violence, et les crimes les plus atroces. Deux jours avant mon arrivée a Kharkoff, dix de ces malheureux avaient épouvanté le pays en assassinant une femme dont ils avaient traversé les membres avec un fer rouge pour lui faire avouer ou était son argent qui consistait en 18 roubles. J'ai vu dans un château des environs de Kharkoff une famille de ces émigrants du Caucase qui ressemblaient à des spectres. Ils s'étaient traînés jusque dans les cours du château et à peine leur avait-on distribué quelques vivres, qu'un des enfants mourut d'épuisement.

J'entre dans ces détails, Monsieur le Maréchal, pour mettre votre Excellence à même d'apprécier l'état de ces provinces, dont la misère présente et ses conséquences nombreuses ne peuvent se comparer à rien de ce qu'une calamité semblable pourrait causer de fâcheux dans des pays administrés comme la France ou l'Allemagne.

Chez les Cosaques du Don, la famine a été complète et un grand nombre de familles ont aussi quitté le pays emmenant avec eux leurs bestiaux. Leur résolution d'émigration a été prise plus tôt que par les habitants, il leur restait encore quelques ressources et des bestiaux. Leur passage par les gouvernements qu'ils traversaient n'a pas été marqué, au dire des autorités, par les mêmes désordres. A Koursk, un seigneur dont les biens sont dans ce gouvernement me disait que des bandes de paysans du Don étaient passés par sa terre, et offraient d'y laisser leurs enfants, donnant 2 et 3 bœufs pour un enfant dont on consentirait à se charger voulant euxmêmes continuer leur route jusque dans les provinces de l'ouest, ou ils espéraient trouver à vivre. Un grand nombre de ces malheureux, épuisés de fatigue et de privations, sont morts en route.

Le gouvernement s'est occupé de son mieux de la répartition de ces émigrants du Caucase et du pays des Cosaques, afin que ces malheureux ne se trouvent pas agglomérés dans un même lieu, et n'y augmentent pas le fléau qu'ils fuyaient. Il faut dire aussi à la louange des populations, que partout ils ont été accueillis avec charité par ceux qui avaient un peu de pain à partager.

En même temps que le gouvernement favorisait les émigrations, qu'il reconnut plus tard un moyen de soulagement peu utile, il prenait d'autres mesures. Là où il y avait des magasins de la couronne, l'ordre fut donné de vendre la farine à un prix modéré et l'on n'a pas hésité à les vider, sauf à les remplir plus tard à des conditions onéreuses. Dans chaque gouvernement et sous la responsabilité du gouverneur, il doit y avoir des magasins de réserve en grains suffisants pour assurer les besoins de

vraisemblable, vu le nombre de colons au Caucase. Ils retournent sur les terres dont ils sont originaires (Koursk). Un seul point noir du tableau dressé par La Ruë semble contestable : ces colons des terres de la Couronne ont sans doute quitté leur ancienne région pour le Caucase à cause du manque de terre et de la misère, mais volontairement et non « conduits de force » vers des terres désertes. 
la population pendant deux ans $\mathrm{s}^{63}$. Mais par suite du mauvais système d'administration, presque jamais ces magasins ne sont approvisionnés, surtout dans le midi, où l'on est plus confiant sur les chances d'une bonne récolte. Plusieurs gouverneurs avaient démontré à tort, qu'il était plus avantageux d'avoir une réserve d'argent ${ }^{64}$. Aussi, lorsque dernièrement, l'on envoya des aides-de-camp de l'empereur dans les divers gouvernements qui souffrent de la disette, pour vérifier les causes et les ressources, ils constatèrent que ces magasins étaient à peu près vides. Le prince Repnin ${ }^{65}$ qui est chef du gouvernement de Poltava, répondit que ses magasins avaient été épuisés lors des guerres de Turquie. Ces magasins n'existaient pas d'avantage avant la guerre de Turquie et le pays fut alors épuisé pour les besoins de l'armée, sans même qu'on eût à disposer de ces réserves qui eussent (été) absorbées également alors, il est vrai, si elles eussent existées.

C'est ici le cas de citer un fait que j'ai pu constater en parcourant les provinces du midi et de l'ouest et que je crois utile de signaler à votre Excellence pour lui faire voir combien la Russie épuise promptement ses ressources lorsqu'elle doit fournir aux besoins de la guerre et combien il lui faut de tonnes pour réparer cet état d'épuisement. On remarque que la disette a été fort augmentée et en partie causée par les trois campagnes de Turquie et de Pologne, dans les provinces qui ont été traversées par les troupes et d'où l'on a tiré les vivres envoyés à l'armée ${ }^{66}$.

63. Les greniers de réserve n'ont pas suffi, mais ils ont certainement limité le nombre de victimes. Le ministère des Apanages donne le compte rendu suivant : « La mauvaise récolte de l'année passée s'est fait surtout ressentir dans les terres et apanages des gouvernements de Penza, Voronège, Saratoff, Smolensk et Pskoff. Il était indispensable d'apporter de grands secours aux paysans, soit en délivrant du grain des magasins, soit en l'achetant, là où les approvisionnements étaient épuisés, et où le transport de grains d'autres magasins offrait trop de difficultés. Il a été délivré des magasins de prévoyance : en seigle -290.667 četv. et en céréales d'été - 104.365 četv. Il a été, en outre, distribué et vendu aux paysans de la Couronne et aux habitants du Don, 3.650 četvert de grains. » (Journal de Saint-Pétersbourg, 10 / 22 avril 1834). Encore deux remarques sur ce sujet : le remplissage annuel des greniers se faisait par prélèvement de 2 četvert de grains par « âme » (revizskaja duša) (art. cit. de l'encyclopédie Brokgauz i Efron, p. 355); il arrivait que les réserves faites fussent détériorées et inutilisables comme cela se produisit dans le gouvernement d'Ekaterinoslav (E. I. Družinina, op. cit., p. 62).

64. Nous avons vu que, depuis 1822 , les gouvernements pouvaient soit constituer des réserves en nature pour les magasins de prévoyance, soit constituer des réserves d'argent. La base de ces dernières était également un versement annuel prélevé sur chaque «âme ». Ayant constaté les limites du système d'approvisionnement en vigueur lors de la disette de 1833, un comité fut instauré en novembre. Ses recommandations sont à l'origine de l'oukase de l'année suivante, imposant dans chaque gouvernement des magasins de prévoyance et une réserve d'argent (art. cit. de l'encyclopédie Brokgauz i Efron, p. 356)

65. Le général prince Nikolaj Grigor'evič Repnin-Volkonskij (1778-1845), gouverneur général de la Petite Russie de 1816 à 1834, aurait fait distribuer de grandes quantités de grain aux paysans souffrant de la disette et avancer des prêts aux propriétaires pour la subsistance de leurs serfs (P. Majkov, in Russkij biografičeskij slovar', Saint-Pétersbourg, 1913, p. 124). Il fut relevé de ses fonctions, accusé de détournement de fonds pendant la famine. Sur cette affaire et, en général, sur la surestimation de l'importance de Repnin par des auteurs français dont Prosper de Barante et Custine, voir Vera Milčina «La Russie en 1839 du marquis de Custine et ses sources contemporaines », Cahiers du Monde russe, 41/1, janvier-mars 2000, p. 160-161.

66. Il s'agit de la guerre de 1828-1829 contre la Turquie qui se déroula sur les fronts des Balkans et du Caucase. La seconde campagne est sans doute l'expédition à Constantinople de1833 et la troisième écrasa la révolution polonaise de 1830-1831. Ces campagnes ont-elles 
En effet, dans les Principautés, les ressources locales ayant été promptement épuisées, il fallut faire arriver des provinces russes voisines les vivres qui manquaient. $0 \mathrm{n}$ a donc mis en réquisition hommes et chevaux pour conduire les grains, les bestiaux et les fourrages de ces provinces. La guerre de Turquie s'étant prolongée, pendant deux campagnes, tout a été consommé, et les hommes et les chevaux enlevés pour les transports ayant presque tous péri de misère, il en est résulté des pertes d'autant plus sensibles, dans la population, qu'elle est encore assez rare dans cette partie de l'empire ${ }^{67}$. On doit reconnaître que les fautes de l'administration militaire et en général les vices de tout système administratif ont contribué à augmenter le mal, et quelques efforts que fasse aujourd'hui le gouvernement, il voit la plupart de ses bonnes intentions paralysées ou annulées par les difficultés qui entravent l'exécution de toutes mesures.

Pour diminuer le mal, on a sursis à l'exécution des mesures pour le payement des impôts arriérés, chez tous les propriétaires sur les terres desquels il a été constaté que la récolte avait manqué68. Dans les mêmes lieux, on a différé le recrutement, on a apporté quelques allègements et quelques délais dans le payement des établissements de crédit; le nouveau cens de la population a été ajourné ; mais en plusieurs endroits on m'a représenté cette mesure comme une perfidie de l'autorité qui distribue ses secours sans tenir compte du changement produit dans la plupart des localités par l'accroissement de la population, alors que le payement des contributions n'était pas exigé, et l'on tient peu compte au gouvernement de n'avoir point fait le recrutement cette année dans les provinces souffrant de la disette ${ }^{69}$, les seigneurs eussent au contraire fourni volontiers deux recrutements à l'avance pour diminuer les consommateurs sur leurs terres.

eu un effet qui se ressent encore sur les céréales disponibles en 1833 et aggravent la famine ? Cela ne nous semble pas certain, bien que nous sachions que la Nouvelle Russie a été mise à contribution pour ravitailler les armées engagées contre les Turcs en 1829 (M. P. Ščerbinin, op. cit., p. 194 ; P. Lacroix, op. cit., vol. IV, p. 392-393) et également lors de l'expédition de 1833 (Marmont, Voyage du maréchal, duc de Raguse..., op. cit., p. 224). Notons aussi que, même en temps de guerre, les prix des céréales, en partie liés à leur abondance ou à leur rareté sur le marché, sont avant tout affectés par les fluctuations des récoltes, d'après l'étude menée par B. N. Mironov : Hlebnye ceny v Rossii za dva stoletija, XVIII-XIX vV. (Les prix des céréales dans la Russie durant deuX siècles, XVIII -XIX ${ }^{e}$ s.), Leningrad, Nauka, 1985, p. 126.

67. Il s'agit des principautés de Moldavie et de Valachie.

68. La remise des impôts arriérés fait partie des mesures traditionnellement mises en œuvre les années de disette (Slovcov, art. cit., p. 490). Ces impôts sont dus par les serfs, les seigneurs en étant exempts.

69. La huitième révision pour le recensement et le recrutement prévoyait initialement la remise des listes pour le $1^{\text {er }}$ août 1833 . Cependant, en raison de la situation des régions du Sud, elle y fut suspendue et eut lieu du $1^{\text {er }}$ septembre 1834 au $1^{\text {er }}$ août 1835 (V. M. Kabuzan, Narody Rossii..., op. cit. p. 68). Ces mêmes gouvernements ont été exemptés de la participation au $98^{\mathrm{e}}$ recrutement par un manifeste impérial du $1^{\text {er }}$ août 1833 stipulant que « Les recrues que les dites provinces auraient été tenues de fournir, seraient considérées comme un arrérage dû par elles. » Mais l'année suivante, un second oukase « accorde à toutes ces provinces remise entière de ce recrutement arriéré, afin de leur donner une nouvelle facilité de rétablir leur ancien état de prospérité. » (Journal de Saint-Pétersbourg, 9/21 août 1834). 
Les grands propriétaires ont donc été aidés par la dispense temporaire d'impôts et d'intérêts d'emprunts, et le prêt leur a été facilité, mais les abus se glissant en grand nombre dans l'application de toutes les mesures du gouvernement supérieur, ces dispositions sont devenues une source de vexations, d'injustices, de dilapidation et de trafic de protection de tous genres. Indépendamment de ce genre de secours, qui a été accordé aux petits propriétaires, le gouvernement a eu la bonne intention de faire distribuer gratuitement à ces derniers, de l'argent, lorsque leur état de détresse a été reconnu et constaté par l'autorité locale. Différentes sommes furent envoyées à cet effet dans les mois de septembre et octobre derniers pour être distribuées, soit à titre de prêt, soit à titre de secours, dans les gouvernements de l'intérieur seulement, les anciennes provinces polonaises n'y eurent aucune part. L'autorité locale, constatait les besoins comme les ressources qui restaient aux propriétaires. Dans l'impossibilité de les secourir tous, le gouvernement n'a fait distribuer des secours qu'aux très petits propriétaires, c'est-à-dire à ceux qui possèdent 50,60, 100 paysans et qui se trouvaient par l'état habituellement précaire de leurs ressources dépourvus de toute espèce de moyens. Mais, à côté de cette bonne volonté du gouvernement à l'égard de ces derniers, sont venus se glisser les difficultés de la répartition et les abus administratifs. Ce sont en grande partie ces petits propriétaires qui composent les administrations secondaires et locales. Ceux-là se sont partagés, même avec une apparence de légalité, au moyen des états fournis et dressés par eux-mêmes, la plus grande partie des fonds de secours.

Les autres petits propriétaires, souffrant comme eux et plus qu'eux, mais n'ayant pas comme eux appui et action dans l'administration locale, n'ont rien eu dans la répartition de ces secours en argent et en grains dont le chiffre est porté à 28000000 de roubles $^{70}$. Ce qui reste disponible après toutes les dilapidations des autorités secondaires, a été appliqué seulement aux paysans de la couronne, et pour eux-mêmes les soins de l'administration ont surtout pour objet d'assurer les semences, passant condamnation sur les souffrances présentes ${ }^{71}$.

Une autre cause qui a contribué à augmenter le mal c'est que malgré la disette des grains, le gouvernement a rigoureusement tenu la main à ce que la ferme des eaux-de-vie, dont le bail rapporte 73000000 de roubles au trésor, remplit toutes les conditions de son marché. Il en résulte tout à la fois une hausse dans le prix du blé et une augmentation de disette par la quantité de blé distillé72. Il n'y a eu interruption

70. Cette estimation est proche de celle de 30 millions de roubles, donnée par l'Encyclopédie Brokgauz i Efron (art. cit., p. 356).

71. Il est peu probable que les paysans de la Couronne n'aient reçu de subsides qu'après que les serfs de petits propriétaires ont été secourus. Le compte rendu cité sur l'activité du ministère des Apanages le dément, puisque ses paysans forment une petite partie des paysans de la Couronne. Le système mis en place était en fait décentralisé, chaque autorité de tutelle publique ou privée assurant des réserves pour les mauvaises années et les répartissant. Il n'y a que pour l'attribution des subsides exceptionnels qu'un tel ordre de priorité aurait pu être adopté, mais cela semble bien incongru.

72. La ferme des alcools, abolie en 1819 pour passer à une perception directe des taxes sur les alcools, a été rétablie en 1827 . Les revenus liés à l'alcool constituent l'une des principales sources de revenus du Trésor, rapportant en général juste un peu moins d'argent que l'ensemble 
forcée des distilleries que là où le prix du blé devenait supérieur à l'amende que doit payer le soustraitant qui ne tient pas les conditions de son marché. Mais presque partout, bien qu'ils aient payé les blés plus cher, les fermiers des eaux-de-vie ont gagné beaucoup d'argent, leurs moyens d'approvisionnement s'étant organisés, lorsqu'ils ont pressenti la disette. Ils ont fait à l'avance des achats plus considérables que le besoin des fermes, et ont ensuite revendu ce blé à ceux qui leur avaient vendu, à des prix triples et quadruples.

Les troupes cantonnées dans les gouvernements souffrant de la disette, ont été partout suffisamment approvisionnées. Il y a des magasins militaires près des lieux de cantonnement et nulle part on ne négligea de les pourvoir avant que la disette se fit beaucoup sentir. De là vient que par suite des précautions prises par l'autorité militaire, les dépenses de ce côté, proportion gardée, ont été moins fortes ${ }^{73}$.

Il faut aussi remarquer que si l'autorité militaire s'est montrée plus prévoyante elle a contribué à diminuer les ressources des paysans en enlevant les blés qui se trouvaient dans les localités voisines des cantonnements. Les troupes colonisées se sont trouvées dans une situation différente et plus favorable, surtout celles des colonies entre le Boug et l'Ingoul, qui ont été formées d'après le système du général de Witte et sont administrées par ses soins ${ }^{74}$. Les colonies de Tchougouieff, dans le gouvernement de Kharkoff, créées d'après un autre mode de colonisation ${ }^{75}$, n'avaient point de réserves suffisantes pour subvenir aux besoins des deux divisions qui les occupent. On a dû envoyer l'une d'elles, celle des cuirassiers dans le

des contributions directes, mais les dépassant cependant certaines années. Ces revenus en 1833 sont de 117 millions sur des entrées totales de 429 millions de roubles assignats, alors que l'année précédente ils étaient de 118 millions sur 451 (W. McKenzie Pintner, Russian economic policy under Nicholas I, Ithaca, NY, Cornell University Press, 1967, p. 76-80). La distillation a donc bien absorbé beaucoup de grain, comme le remarque La Ruë. Il est arrivé que la distillation d'alcool fût interdite certaines années de disette, une première fois en 1748 et une seconde, en 1792, dans le gouvernement de Vitepsk (Slovcov, art. cit., p. 492).

73. L'armée a non seulement assuré le ravitaillement de ses troupes, mais elle a pu mettre à la disposition de diverses administrations des réserves qu'elle avait constituées : ainsi à SaintPétersbourg, où elle vendit aux habitants pauvres du blé au prix standard, dans la seconde moitié de 1833 et à Moscou en 1834 (I. F. Dubrovin, « Materijaly i čerty k biografii Nikolaja I... », art. cit., p. 353).

74. Comme nous l'avons vu, il n'y a pas à proprement parler de «système de Witte » qui s'opposerait à celui d'Arakčeev. Mais, dans les colonies qu'il a créées à partir de 1821 dans le gouvernement de Kherson, dont il est question ici, Witte a dès le départ imposé aux paysans colonisés de nourrir les 400 personnes et 200 chevaux venus organiser la colonie et prévu que les nouveaux colons fourniraient, dans un délai de trois ans, l'approvisionnement de toute la division de cavalerie colonisée, ce qui fut fait. Arakčeev essaya d'obtenir de pareils résultats dans les autres colonies (A. N. Petrov, Graf Arakčeev ..., op. cit., p. 95).

75. Tchougouev, ville située à $40 \mathrm{~km}$ au sud-est de Kharkov, est devenue en 1817 le centre d'une colonie militaire, établie sur les terres des cosaques du Boug, incorporés dans une division de uhlans colonisée. Cette opération, à laquelle étaient opposés ces cosaques, a été menée sous la direction du lieutenant général de Witte, à la tête de cette division, et conformément aux instructions d'Arakčeev (P. P. Evstaf'ev, Vosstanie voennyh poseljan Novgordoskoj gubernii v 1831 g. (La révolte des colons militaires du gouvernement de Novgorod en 1831), Moscou, 1934 , p. 62 ; P. P. Karcov, « O voennyh poselenijah... », art. cit., t. 207, p. 89). À l'époque de la famine de 1833, Witte était inspecteur des colonies de cavalerie, tandis qu'Arakčeev avait été écarté de la direction des colonies en 1826. 
gouvernement de Tamboff. Les frais nécessités par ce déplacement et l'entretien de cette seule division dans ce gouvernement où les vivres et les fourrages étaient aussi fort rares, se sont élevés à 1400000 roubles.

Au lieu d'un résultat aussi onéreux, les colonies du général de Witte se sont trouvées avoir des approvisionnements suffisants en grains et en fourrages pour nourrir quatre divisions de cavalerie, environ 25000 hommes y compris les réserves de cantonnistes ; venir au secours de 400000 âmes de population et de 450000 têtes de bétail. Malgré cette consommation forcée pendant huit mois, ces colonies ont encore en réserve près de 200000 chetwerts de grains et de foin, mais dans une proportion moindre, c'est-à-dire de quoi faire vivre les quatre divisions de cavalerie pendant dix-huit mois ${ }^{76}$.

Ces résultats avantageux parlent en faveur du système de colonisation du général de Witte. Je l'ai étudié avec soin sur les lieux et j'aurai l'honneur de soumettre à votre Excellence les renseignements que j'ai recueillis et les observations que j'ai été à même de faire sur cette importante organisation des colonies militaires du midi de la Russie.

On a déjà pu voir par quelques uns des détails qui précèdent combien l'importation est difficile dans la plupart des gouvernements qui souffrent de la disette. Pour mieux fixer les chances de spéculation que présenterait au commerce la prolongation de cet état de disette, que la chaleur anticipée et la sécheresse continuelle de ce printemps rendent possible, je joindrai à ce rapport l'état de mouvement des prix des grains à Odessa depuis six mois.

\section{Prix moyens des grains à Odessa, par semaine, depuis le $1^{\text {er }}$ Octobre 1833,} jusqu'au 30 avril/12 mai1834, en roubles et par chetwert

\section{Octobre 1833}

\begin{tabular}{|l|c|c|c|c|c|c|c|c|}
\cline { 2 - 9 } \multicolumn{1}{c|}{} & \multicolumn{2}{c|}{$\mathbf{1}$ semaine } & \multicolumn{2}{c|}{ 2 semaine } & \multicolumn{2}{c|}{ 3 semaine } & \multicolumn{2}{c|}{ 4 semaine } \\
\cline { 2 - 9 } \multicolumn{1}{c|}{} & $\begin{array}{c}\mathbf{1} \text { re } \\
\text { qualité }\end{array}$ & $\begin{array}{c}\text { qualité } \\
\text { inférieure }\end{array}$ & $\mathbf{1}$ q. & q.i. & $\mathbf{1}$ q. & q.i. & 1 q. & q.i. \\
\hline blé tendre & 26 & 24 & 26 & 24 & 26 & 24 & 26 & 24 \\
\hline blé dur & 35 & 29 & 35 & 29 & 35 & 30 & 35 & 30 \\
\hline seigle & 28 & 25 & -- & - & - & - & - & - \\
\hline orge & 17 & $16,1 / 2$ & -- & - & - & - & - & - \\
\hline avoine & 13 & 12 & -- & - & - & - & - & - \\
\hline
\end{tabular}

Exporté en octobre pour l'étranger 11200 chetw. blé

ports russes 65350 blés et autres grains

76. L'action du comte de Witte est exposée dans un rapport au ministre de la Guerre du 15 / 27 juin 1834, soumis ensuite à Nicolas I ${ }^{e r}$ qui exprime sa grande satisfaction à l'intéressé. Le rescrit de félicitation du 7 / 29 juillet 1834 reprend les principaux points exposés ici et propose des récompenses au comte de Witte et aux fonctionnaires ayant pris part à cette action et que ce dernier désignera (P. Lacroix, op. cit., vol. VI, p. 421-422). 


\section{Novembre}

\begin{tabular}{|l|c|c|c|c|c|c|c|c|}
\cline { 2 - 9 } \multicolumn{1}{c|}{} & \multicolumn{2}{c|}{$\mathbf{1}$ semaine } & \multicolumn{2}{c|}{ 2 semaine } & \multicolumn{2}{c|}{3 semaine } & \multicolumn{2}{c|}{4 semaine } \\
\cline { 2 - 9 } \multicolumn{1}{c|}{} & $\begin{array}{c}\mathbf{1}^{\text {re }} \\
\text { qualité }\end{array}$ & $\begin{array}{c}\text { qualité } \\
\text { inférieure }\end{array}$ & $\mathbf{1}$ q. & q.i. & $\mathbf{1}$ q. & q.i. & $\mathbf{1}$ q. & q.i. \\
\hline blé tendre & 26 & 24 & 26 & 24 & 26 & 24 & $261 / 2$ & 26 \\
\hline blé dur & 35 & 30 & 35 & 31 & 35 & 30 & 37 & 32 \\
\hline seigle & 25 & 25 & - & - & - & - & - & - \\
\hline orge & 18 & 18 & - & - & - & - & - & - \\
\hline avoine & 15 & 14 & - & - & - & - & - & - \\
\hline
\end{tabular}

En novembre point d'exportation pour l'étranger.

$\begin{array}{ccc}\text { dépôts sur plan } & 78000 \text { chetwerts blé tendre } \\ & 17700 & \begin{array}{c}\text { dur } \\ \text { seigle }\end{array} \\ ------- & \text { orge } \\ 10000 & \text { avoine } \\ 15000 & \end{array}$

Décembre

\begin{tabular}{|l|l|l|l|l|l|l|l|l|}
\hline blé tendre & 28 & 26 & 27 & 26 & 28 & 26 & 28 & 26 \\
\hline blé dur & 37 & 32 & 37 & 32 & 35 & 32 & 38 & 32 \\
\hline
\end{tabular}

Point d'achat dans les autres grains et point d'exportation à l'étranger

Dépôt à Odessa à la finde l'année 85000 chetwerts blé tendre

\begin{tabular}{ll}
20000 & \multicolumn{1}{c}{ dur } \\
seigle \\
10000 & orge \\
12000 & avoine
\end{tabular}

\section{Janvier 1834}

\begin{tabular}{|l|c|c|c|c|c|c|c|c|}
\hline blé tendre & 26 & $241 / 2$ & 26 & $241 / 2$ & 25 & $231 / 2$ & 25 & 23 \\
\hline blé dur & 22 & 29 & 32 & 28 & 28 & 26 & 33 & 29 \\
\hline seigle & 25 & $241 / 2$ & 25 & 25 & $241 / 2$ & 24 & $241 / 2$ & 24 \\
\hline orge & $171 / 2$ & 17 & 21 & 17 & $161 / 2$ & 12 & $161 / 2$ & 16 \\
\hline avoine & 6 & $151 / 2$ & 16 & 15 & 15 & 15 & 15 & $141 / 2$ \\
\hline
\end{tabular}

Port gelé. Dépôt en janvier : 85000 chetwerts blé tendre

\begin{aligned} 90000 & \multicolumn{1}{c}{ dur } \\ 2500 & seigle \\ 10000 & orge \\ 12000 & avoine \end{aligned}


Février

\begin{tabular}{|l|c|c|c|c|c|c|c|c|}
\cline { 2 - 9 } \multicolumn{1}{c|}{} & \multicolumn{2}{c|}{$\mathbf{1}$ semaine } & \multicolumn{2}{c|}{ 2 semaine } & \multicolumn{2}{c|}{ 3 semaine } & \multicolumn{2}{c|}{ 4 semaine } \\
\cline { 2 - 9 } \multicolumn{1}{c|}{} & $\begin{array}{c}\mathbf{1} \text { re } \\
\text { qualité }\end{array}$ & $\begin{array}{c}\text { qualité } \\
\text { inférieure }\end{array}$ & $\mathbf{1} \mathbf{~ q .}$ & $\mathbf{q . i .}$ & $\mathbf{1 ~ q .}$ & $\mathbf{q . i .}$ & $\mathbf{1} \mathbf{q .}$ & $\mathbf{q . i .}$ \\
\hline blé tendre & 25 & $231 / 2$ & $241 / 2$ & $231 / 2$ & 24 & 23 & 23 & 21 \\
\hline blé dur & 23 & 29 & 33 & 30 & 23 & 28 & 32 & 27 \\
\hline seigle & $241 / 2$ & 24 & $241 / 2$ & 24 & 24 & $231 / 2$ & 24 & 23 \\
\hline orge & $161 / 2$ & 16 & $151 / 2$ & $141 / 2$ & $151 / 2$ & $141 / 2$ & 15 & $141 / 2$ \\
\hline avoine & 15 & $141 / 2$ & 14 & $131 / 2$ & 14 & 13 & $131 / 2$ & 13 \\
\hline
\end{tabular}

Dépôts en ville à la fin de février : 80000 chetwerts blé tendre

\begin{aligned} 18000 & \multicolumn{1}{c}{ dur } \\ 1500 & seigle \\ 10000 & orge \\ 10000 & avoine \end{aligned}

N.B. Ces grains arrivent du Danube.

\section{Mars}

\begin{tabular}{|l|l|l|l|l|l|l|l|l|}
\hline blé tendre & 22 & 19 & 22 & 19 & 22 & 20 & 231 & 19 \\
\hline blé dur & 31 & 26 & 30 & 27 & 30 & 26 & 28 & 25 \\
\hline seigle & 23 & $221 / 2$ & 23 & $221 / 2$ & 23 & 22 & 22 & 21 \\
\hline orge & 16 & 15 & 16 & $151 / 2$ & 16 & 15 & 15 & 12 \\
\hline avoine & $131 / 2$ & 13 & $131 / 2$ & 13 & 13 & 13 & $121 / 2$ & 12 \\
\hline
\end{tabular}

Exportation seulement pour les ports russe ; la quantité n'est pas indiquée

Dépôts en ville à fin mars : 60000 chetwerts blé tendre

\begin{aligned} 15000 & \multicolumn{1}{c}{ dur } \\ 2500 & seigle \\ 12000 & orge \\ 8000 & avoine \end{aligned}

N.B. Beaucoup de ces grains arrivent du Danube et du Bosphore

\section{Avril}

\begin{tabular}{|l|l|l|l|l|l|l|l|l|}
\hline blé tendre & 20 & $181 / 2$ & 20 & 18 & $191 / 2$ & 18 & 21 & $181 / 2$ \\
\hline blé dur & 27 & 24 & 27 & 24 & 26 & 23 & 27 & 24 \\
\hline seigle & 21 & 20 & 21 & 20 & $191 / 2$ & 19 & 18 & 22 \\
\hline orge & 13 & 12 & 13 & 12 & 14 & 8 & 15 & 12 \\
\hline avoine & 13 & 12 & 13 & 12 & 14 & 13 & 15 & 14 \\
\hline
\end{tabular}

Exportation continue du Danube; les quantités ne sont pas indiquées.

Exportation pour ports russes, ; les quantités ne sont pas indiquées.

Dépôts à la fin d'avril sur plan : 40000 chetw. blé tendre

\begin{aligned} & 15000 \multicolumn{1}{c}{ dur } \\ &-8000 seigle \\ & 5000 orge \\ & avoine \end{aligned}


En étudiant ce tableau, on sera amené à faire la remarque suivante : que les prix des grains sur le marché d'Odessa n'indiquent pas les besoins dans l'intérieur du pays $^{77}$. Les moyens de transport depuis octobre jusqu'au mois d'avril étant nuls, ou très difficiles ${ }^{78}$ et si même les communications étaient plus ouvertes, il est douteux que des spéculateurs particuliers d'Odessa hasardassent des entreprises de fournitures dans l'intérieur, l'extrême misère du peuple rendant une prompte et sûre réalisation impossible ${ }^{79}$. C'est pourquoi l'on a vu les prix baisser à Odessa à l'époque même des plus grands besoins à l'intérieur.

Au moment où je me trouvais dans le gouvernement de Koursk et de Kharkoff, qui n'ont pas été les plus maltraités, c'est-à-dire à la fin d'avril et au commencement du mai, le chetwert de blé se payait 25 roubles, qui vaut ordinairement 5 roubles.

Depuis Orel, la mesure d'avoine ordinairement à 2 roubles se paye de 12 roubles par le gouvernement pour la cavalerie ${ }^{80}$, et le poud de foin habituellement à 20 copecks est à 1 rouble 40 copecks. Je tiens ces renseignements de plusieurs officiers des deux divisions de dragons qui sont échelonnées depuis Orel jusqu'aux environs de Kharkoff. Plus au midi, encore à Krementchoug, sur le Dnieper, un mauvais pain noir qui vaut ordinairement quatre copecks, se payait à l'époque de mon passage 28 copecks. Dans la mer d'Azof les prix sont encore plus élevés. À Taganrog les blés ont été au mois de novembre à 80 roubles le chetwert; ils sont encore à 36 et à 38 roubles. Et malgré les secours que le comte de Woronzoff a distribués dans ses gouvernements j'ai vu une si grande misère parmi les Tartares de la Crimée, que des familles entières étaient mortes de faim.

77. Parmi ces prix, les plus significatifs pour le marché russe d'alors sont ceux du seigle et de l'avoine, ces céréales occupant la première place parmi les cultures du pays. La production de blé (froment) prend cependant de l'importance à partir du XIXe siècle dans la région économique des steppes (stepnoj rajon) qui comprend les gouvernements de Bessarabie, Ekaterinoslav, Tauride et Kherson. La stabilité des prix du seigle en 1833-1834 est bien attestée dans la ville de Kherson, la plus proche sur laquelle nous ayons les données de Mironov qui suit les prix annuels moyens. Cependant, en replaçant ces prix dans une période plus longue, on voit qu'ils avaient beaucoup augmenté ces années-là : 18 kopecks/or par livre en 1829, 33 en 1830, 38 en 1831, 29 en 1832, 59 en 1833, 59 en 1834, 39 en 1835, 17 en 1836 (B. N. Mironov, op. cit., p. 239). Pour leur part, les prix annuels de l'avoine ont en moyenne beaucoup augmenté dès 1833 et ont continué en 1834, selon l'étude de Mironov : 38 kopecks/or par livre en 1829, 37 en 1830, 35 en 1831, 27 en 1832, 58 en 1833, 84 en 1834, 68 en 1835, 38 en 1836 (ibid, p. 260).

78. Si les transports par voie d'eau sont effectivement arrêtés l'hiver, il n'en est pas de même de ceux par voie de terre. Dulov donne un aperçu du roulage et indique qu'au milieu du XIX ${ }^{\mathrm{e}}$ siècle 800000 personnes y participaient et 3000000 l'hiver (op. cit., p. 128-129).

79. Il semble que La Ruë sous-estime le réseau traditionnel de foires et de marchands ambulants les animant encore en place en Russie dans ce premier tiers du XIX ${ }^{\mathrm{e}}$ siècle et y jouant le premier rôle, loin devant les autres activités commerciales (voir par exemple, B. N. Mironov, Vnutrinnij rynok Rossii vo vtoroj polovine XVIII-pervoj polovine XIX V. (Le marché intérieur de la Russie, deuxième moitié du XVIII - première moitié du XIX siècle), Leningrad, Nauka, 1981,p. 67-72). Mais nous ne connaissons pas la réactivité de ce réseau dans des circonstances exceptionnelles telles que celles de 1833-1834.

80. Ces variations observées au printemps 1834 et donnant des prix multipliés par six sont plus grandes que celles observées pour les prix moyens pendant les années 1833 et 1834, qui, eux, sont multipliés par 2,33 (V. N. Mironov, Hlebnye ceny..., op. cit., p. 257). 
Cependant il n'y a point de spéculateurs qui veulent profiter de la grande marge que présentent ces derniers marchés. Ils pensent encore moins aux places éloignées de la côte, où les besoins sont encore plus urgents. À Tiflis par exemple le tchetwert de blé se paye en ce moment 80 roubles.

On ne s'attend pas à Odessa à une importation de l'étranger, à l'exception de quelques petits chargements du Danube. Enfin les besoins du pays n'animeront guère les marchés de grains, à moins que le gouvernement n'entreprenne quelque grande spéculation.

Les données ci-dessus résultent des renseignements que j'ai recueillis près des consuls et des principaux négociants d'Odessa. Ces réflexions répondent à la question contenue dans mes instructions sur la libre importation future des céréales dans les provinces méridionales de l'empire.

Des détails contenus dans ce rapport l'on peut conclure que la Russie méridionale se rétablira difficilement et très lentement de l'épuisement causé par la disette de 1833. Les chaleurs anticipées et le manque de pluie donnent encore les plus sérieuses inquiétudes sur la récolte de cette année. Pendant mon séjour à Odessa et en Crimée vers la fin de mai, on s'accordait à dire que si cette sécheresse durait encore dix jours, tous les foins seraient perdus et les steppes brûlées ${ }^{81}$. Et l'on a vu que non seulement les semailles avaient été insuffisantes, mais encore que la culture sera pendant longtemps empêchée par l'immense destruction des bestiaux de labour.

En relisant mes instructions, je crois que les détails contenus dans ce rapport fournissent tous les renseignements qui m'étaient demandés.

Je suis avec un profond respect, Monsieur le maréchal, de votre Excellence le très humble et très obéissant serviteur.

Le baron de La Ruë

Chef de b[ataill]on, aide-de-camp de son Excellence Maréchal Maison.

Etc..,Etc...

P.S. Ce rapport mettra votre Excellence dans le cas de pressentir ce que vont être les embarras de l'administration et les souffrances de la population de ces mêmes gouvernements de 1a Russie méridionale que l'extrême sécheresse de ce printemps prive encore de récoltes pour cette année. En revenant de Turquie j'ai parcouru plusieurs de ces provinces. Dans les moins maltraitées on récoltera un peu de blé. Tous les autres grains et les foins ont été entièrement brûlés.

Un fait digne aussi d'être constaté, c'est que le gouvernement vient de renouveler le bail de la ferme des eaux-de-vie qui expirait cette année avec une augmentation de 23 millions de roubles consentie par les fermiers. Dans les circonstances actuelles, cette augmentation prouve tout à la fois les besoins du trésor et les bénéfices calamiteux des fermiers.

81. Les récoltes de 1834 ont finalement été bonnes dans l'ensemble, sauf dans les gouvernements de Riazan et de Tambov. En Crimée, où elle s'annonce meilleure que l'année précédente, la récolte n'est cependant pas suffisante pour couvrir les besoins de ses habitants (Journal de Saint-Pétersbourg, 26 juillet / 7 août 1834). 


\section{II \\ Rapport sur les colonies militaires*}

\section{Monsieur le Maréchal,}

Avant de mettre sous les yeux de votre Excellence les renseignements que j'ai réunis sur les colonies militaires de cavalerie, en parcourant ces établissements dans les gouvernements du midi de la Russie, je crois nécessaire de rappeler les motifs qui ont déterminé l'empereur Alexandre à adopter le système des colonies militaires, les essais qui ont été tentés, les dépenses qu'ils ont occasionnées et les divers résultats qu'ils ont produits.

Il est aisé de concevoir quand on jette un coup d'œil sur l'état de l'armée russe, qu'un recrutement est une opération longue et difficile dans un pays aussi étendu, que toutes les ressources pour soutenir une guerre prolongée sont éloignées des endroits où elles peuvent être mises en action, que l'administration extrêmement vicieuse est une source de vexations intolérables pour le pays, que l'éparpillement des troupes est une nécessité à cause des seules distances et un inconvénient pour l'instruction, que le sort du soldat privé des liens de famille, de patrie en quelque sorte, d'asile à la fin de son service, est fort malheureux, que cette situation le rend une charge pour le pays, enfin que les finances souffrent d'entretenir une armée aussi nombreuse et que cependant la diminuer est impossible.

Ces considérations et d'autres d'un ordre plus élevé, déterminèrent l'établissement des colonies militaires. On ne peut douter que l'empereur Alexandre n'ait entrevu un moyen de donner plus de forces à son pays dans le système qu'il a adopté pour l'exécution de ce projet ${ }^{82}$. L'éducation des fils de soldats qui était intimement liée à ce plan, indiquait assez qu'il voulait avancer la marche de la civilisation $^{83}$ et se créer une classe intermédiaire dont le besoin se fait sentir tous les jours davantage en Russie. Les préjugés qu'il a sapés par cette institution, ceux religieux qu'il a attaqués pour ramener à l'unité les dissidents grecs qu'il a particulièrement soumis $^{84}$, les constructions, les chemins, les communications ouvertes, tout annonce qu'il avait des intentions ultérieures et que ce n'était pas seulement des places d'armes qu'il voulait fonder. La politique ne lui permettait sans doute pas de

* En romain : le texte commun à l'article du Spectateur militaire de 1828 et au rapport manuscrit de 1834. En italique : le texte modifié ou ajouté dans le rapport manuscrit.

Nous n'avons pas tenu compte de légères modifications de forme entre les deux versions .

82. Comme la plupart des auteurs occidentaux de son époque, La Ruë attribue bien le projet de colonies militaires à Alexandre Irr personnellement. Mais, Tanski fait d'Arakčeev l'instigateur de ce projet (J. Tanski, Tableau statistique, politique et moral..., op. cit., p. 119), comme le feront d'autres auteurs.

83. Tous les auteurs occidentaux contemporains de La Ruë sont convaincus de l'intention civilisatrice d'Alexandre Ir. Richard Pipes met à nouveau en exergue ce point de vue, rompant avec l'historiographie traditionnelle qui rattachait les colonies militaires à la phase réactionnaire du règne d'Alexandre Ier (R. Pipes, « The Russian military colonies... », art. cit., p. 205, 210-211).

84. Il s'agit des vieux-croyants de la région de Novgrorod. Voir par exemple « Vospominanija M. O. Borozdina » (Les souvenirs de M. O. Borozdin), in Graf Arakčeev i voennye poselenija..., op. cit., p. 2. 
faire connaître toute sa pensée à cet égard et elle ne paraît pas avoir été devinée par tous ceux qui ont été chargés de mettre à exécution cette vaste conception ${ }^{85}$.

Le but avoué de ce projet, qui dès son origine a trouvé dans les classes élevées de la société une opposition dont il est facile de se faire une juste idée 86 , était :

1 - de diminuer la dépense de l'armée ;

2 - d'en assurer le recrutement par le mouvement de la population et sans le concours du gouvernement ;

3 - de conserver les bras à l'agriculture en temps de paix et de tenir les troupes toujours prêtes à marcher ;

4 - de donner un asile aux militaires inférieurs et inutiles.

Pour obtenir ces avantages et assurer le succès des colonies militaires, le choix des provinces où elles devaient être établies était le point capital et celui qui a été fait pour les colonies d'infanterie n'a pas été heureux.

Le comte Aracktchieff investi de toute la confiance de l'empereur Alexandre s'est conduit dans cette circonstance plus en courtisan qu'en administrateur habile et prévoyant ; craignant de s'éloigner de la cour, de perdre par là l'influence qu'il exerçait et chargé de mettre à exécution ce vaste projet dont la première pensée appartient à l'impératrice Cathérine, et dont l'empereur Alexandre avait rédigé luimême les règlements en 1808 , Aracktchieff désigna pour la colonisation des trois divisions de grenadiers les gouvernements de Pétersbourg et de Novogorod ${ }^{87}$.

La première division qui en quelque sorte a servi de modèle a été établie sur le Wolkof, qui fait partie du grand système de navigation qui amène à Pétersbourg tous les produits de la Russie méridionale. Ses rives peu habitées, incultes, malsaines n'offraient ni colons agricoles, cette faible population étant adonnée au commerce, ni établissements primitifs, ni communications, rien enfin de ce qui aurait pu en déterminer le choix si ce n'est la proximité de la capitale ${ }^{88}$.

85. Karcov montre bien qu'au départ l'opération était secrète et que le déménagement du $1^{\text {er }}$ bataillon s'est effectué pour le motif officiel du manque de caserne à Saint-Pétersbourg. P. Karcov, « O voennyh poselenijah... », art. cit., t. 206, p. 146-147.

86. Dans l'entourage proche de l'empereur, le haut commandement militaire (Barclay de Tolly, Dibič) y était particulièrement hostile. Voir par exemple les documents exprimant ses préventions à l'égard de cette institution reproduits par P. P. Evstaf'ev, Vosstanie voennyh poseljan..., op. cit., p. 45-50.

87. Expliquer le choix de la région de Novgorod par l'attitude d'Arakčeev, courtisan ne voulant pas perdre son influence, reflète sans doute ce qui se disait sur ce personnage. Cependant, deux faits viennent contredire cette explication : le premier établissement de colonies a eu lieu en 1809 dans la région de Moghilev, dans l'actuelle Biélorussie, et donc loin de la capitale ; le domaine personnel d'Arakčeev se trouvait sur le Volkhov, à Gruzino, et c'est précisément ce domaine, dont Alexandre Ier admirait l'ordre, qui a servi de modèle aux colonies militaires. Leur implantation à proximité de Gruzino les plaçait d'emblée sous le contrôle étroit d'Arakčeev, ce qui était conforme aux vœux d'Alexandre I'r.

88. La région de Novgorod était certes marécageuse, mais pas déserte. Ce sont bien les paysans locaux, de 19 villages pour le $1^{\text {er }}$ bataillon colonisé en 1816 (Petrov, p. 91), de 56 villages dans les colonies établies en 1818 (P. P. Evstaf'ev, Vosstanie voennyh poseljan..., op. cit., p. 63-64), qui ont été incorporés dans ces colonies. Cette région a bien une tradition commerciale, mais il semble que les paysans de la région qui avaient l'habitude de commercialiser de l'avoine, du lin et du bois de chauffage ont continué à le faire et, qu'en l'espace de cinq ans après leur enrôlement dans les colonies, le volume de leurs transactions avait doublé (P. Karcov, art. cit., t. 207, p. 111- 
Les six régiments colonisés n'ont pas reçu plus de cinq à six mille colons ${ }^{89}$, et en Autriche où ce système a eu un succès complet, on compte qu'il faut que la population soit quinze fois plus forte que le nombre de soldats que le pays doit fournir. $J$ 'ai visité il y a trois ans les régiments frontières établis dans la Croatie, le Banat de Temeswar et la Transylvanie. Je pourrais fournir sur le système de colonisation suivi par l'Autriche des notes détaillées si le ministre le désire.

En Russie et dans la localité désignée tout était donc à faire, à créer, constructions, défrichements, marais à dessécher, canaux à percer, etc... Des sommes immenses y ont été employées et la population ne se trouvant pas en rapport avec les besoins des régiments on n'en a retiré d'autres avantages que le défrichement d'étendues de terre assez considérables, avantages qui ne peuvent même pas être donnés comme compensation des belles forêts situées à peu de distance de SaintPétersbourg et sur une rivière qui établit entre cette ville et Novogorod une communication facile ${ }^{90}$. En calculant les dépenses occasionnées par les constructions, les défrichements, les dessèchements, l'achat des bestiaux fournis aux habitants, la remise de l'impôt (obrok), la nourriture des habitants pendant plusieurs années, on peut sans exagération porter les frais de colonisation d'un régiment d'infanterie à 5 millions de roubles ${ }^{91}$. En supposant que la population ait pu par la suite nourrir la troupe, il en résulterait pour l'état une économie de 30 mille roubles à raison de 2000 hommes au prix de 15 roubles par an, qui serait l'intérêt à moins de $1 \%$ des 5 millions de capitaux qui y ont été enfouis.

Les deux autres divisions de grenadiers dont les $3^{\mathrm{e}}$ bataillons seulement étaient colonisés dans les environs de Staraia Roussa ${ }^{92}$, sur les bords du lac Ilmen n'étaient pas plus heureusement placées et ne donnaient pas des résultats plus satisfaisants.

112). Von Bradke considère aussi que le choix de la région de Novgorod n'était pas heureux en raison des marais, des forêts et de la nature du sol demandant un immense travail pour sa mise en valeur («Avtobiografičeskie zapiski ... », art. cit., t. I, p. 51).

89. Le chiffre donné ici paraît modeste. Pour l'ensemble des colonies de la région de Novgorod, Karcov parle d'une population de 100000 personnes (art. cit., t. 206, p. 169).

90. D'immenses travaux de construction (casernements et routes) et d'assèchement de marais furent entrepris A. A. Ejler parle de 30000 ouvriers employés à des travaux de construction l'été 1824. Et il mentionne les grandes quantités de bois tirées des immenses forêts de la région («Zapiski A. A. Ejlera (Notes de A. A. Ejler), Russkij arhiv, II, 1880, p. 375, 377). Toutefois, La Ruë exagère leur proximité de Saint-Pétersbourg qui rendrait leur perte plus dommageable.

91. Il s'agit de roubles assignats, dont la valeur est environ le quart des roubles argent. Cette estimation n'est pas très éloignée de celle de von Bradke, qui avance le chiffre de 4 millions de roubles assignats pour chaque régiment : trois millions pour la construction des bâtiments et des routes, un million pour le bétail, les instruments agricoles, les semences nécessaires à la mise en valeur initiale («Avtobiografičeskie zapiski ... », art. cit., t. I, p. 52).

92. Les paysans enrôlés dans les colonies de Staraia Roussa ont conservé leurs maisons individuelles traditionnelles et n'eurent pas, comme ceux des colonies sur le Volkhov, à emménager dans de nouvelles grandes bâtisses ( svjazi) prévues pour quatre maîtres-colons, leurs familles et les soldats cantonnés chez eux (P. Karcov, art. cit., t. 207, p. 83-84). 
En résumé les colonies militaires d'infanterie présentaient tant de vices dans leur système d'établissement, qu'il a été d'une bonne politique de les dissoudre ${ }^{93}$ et la révolte qui éclata dans celle de Novogorod était l'expression d'un mécontentement qui datait de l'origine des colonies. Soldats et paysans colons firent cause commune et saisirent comme occasion favorable les troubles causés par le choléra et qui leur firent croire que Saint-Pétersbourg était en pleine révolution. En trois heures la révolte se propagea sur une étendue de soixante verstes et dura 48 heures $^{94}$. 80 officiers ont été massacrés avec des détails de cruautés inouïes, plusieurs de leurs femmes éventrées et les enfants portés au haut des bayonnettes ; plus de cent autres personnes employées dans la colonie et quelques seigneurs des environs périrent soupçonnés d'être partisans du système de colonisation ${ }^{95}$. Le général Aracktchieff dont le château est dans les environs, averti par un domestique, feignit d'attacher peu d'importance au mouvement, fit même conduire un cheval à la porte de son parc et sortit comme pour aller au devant des insurgés, mais il se sauva dans la campagne où il erra pendant deux jours sans prendre de nourriture et sans que personne chez lui sut ce qu'il était devenu. Les soldats le cherchèrent pour le massacrer, mais ne détruisirent rien dans son habitation ${ }^{96}$. Des officiers se couchèrent dans des champs, d'autres parvinrent à s'enfuir... L'empereur averti, envoya le général Orloff, qui arriva seul au milieu des révoltés, les pérora et parvint à les calmer ${ }^{97}$. L'empereur Nicolas le suivit, il arriva seul aussi au milieu d'eux, à son aspect ils se soumirent tous. L'empereur leur dit qu'il ne voulait d'eux qu'une

93. Nous avons vu qu'il s'agit d'une profonde transformation en districts de soldats laboureurs, plutôt que d'une suppression à proprement parler. L'administration militaire des terres formant jusqu'en 1831 les colonies militaires de Novgorod et de Staraia Roussa n'est abolie qu'en 1856. La Ruë n'est pas le seul à s'exprimer ainsi, A. K. Gribbe, dans ses souvenirs, considère aussi que 1832 marque la fin de l'existence des colonies de Novgorod (« Novgorodskie voennye poselenija. Vospominanija A. K. Gribbe » (Les colonies militaires de Novgorod, Souvenirs de A. K. Gribbe), Russkaja starina, t. 45, 1885, p. 151).

94. Cette révolte, partie le 11 juillet 1831 de Staraia Roussa, gagne l'ensemble des colonies du Nord. Elle est effectivement liée à l'épidémie de choléra qui a atteint Saint-Pétersbourg et à l'incompréhension des mesures de quarantaine imposées aux colonies militaires. Prenant cette épidémie pour un empoisonnement collectif, perpétré par l'encadrement militaire acheté par les insurgés polonais, ouvriers des chantiers de construction, paysans, soldats et quelques sousofficiers massacrent médecins, officiers et sous-officiers dont il n'avaient jamais supporté la discipline de fer qu'ils leur imposaient et, pour certains, les détournements financiers à leurs dépens (voir les souvenirs de M. O. Borozdin, in Graf Arakčeev..., op. cit., p. 10-15 ; Alan D. Ferguson, « The Russian military settlements... », art. cit., p. 114-115).

95. Lykošin donne le nombre de plus de cent officiers et médecins tués par les colons insurgés ou morts des suites de mauvais traitements («Voennye poselenija », art. cit.,p. 610), tandis que Ferguson avance celui de près de deux cents « officiers, médecins et officiels » (A.D. Ferguson, art .cit., p. 114).

96. D’après Borozdin, Arakčeev, effectivement recherché par les soldats insurgés, aurait trouvé refuge au monastère Saint-Georges, proche de Novgorod (in Graf Arakčeev ..., op. cit., p. 15).

97. Ce récit est un peu simpliste. Le général Andrej Fedorovič Orlov (1787-1862), homme de confiance de Nicolas Irer, arrive dans les colonies alors que l'ordre y a déjà été rétabli par le général Kleinmichel, commandant en chef des colonies de Novgorod (P. Lacroix, op. cit. , vol. V,p. 486). 
chose, c'est que chacun rentrât chez lui pendant la nuit ${ }^{98}$. On se saisit des murmures. Environ cent sous-officiers furent suppliciés, d'autres envoyés en Sibérie. Ce mouvement insurrectionnel coûta la vie à près de 400 personnes massacrées par les révoltés ou mises à mort par l'autorité ${ }^{99}$, mais de ce mouvement date la suppression des colonies d'infanterie.

Je m'étendrai davantage sur les colonies de cavalerie dont la direction a été confiée au comte de Witte, homme de beaucoup d'esprit, bon administrateur et qui favorisé par les localités et envisageant les établissements sous un point de vue tout à fait différent que le comte Aracktchieff, paraît avoir atteint le but qu'on se proposait sous le rapport de l'instruction, du recrutement et de l'économie ${ }^{100}$.

Deux divisions, une de lanciers, l'autre de cuirassiers ont été colonisées à Tchougouieff dans le gouvernement de Kharkoff. La première sur le plan modifié du comte Aracktchieff, celle des cuirassiers d'après le système du comte de Witte ${ }^{101}$.

Depuis la suppression des colonies d'infanterie, l'empereur avait eu l'intention d'abolir aussi celles de cavalerie de Tchougouieff, mais tout récemment il les a placées sous le commandement du général de Witte, qui s'occupe en ce moment de leur réorganisation ${ }^{102}$. La division de cuirassiers a été momentanément envoyée en

98. De même, le récit de La Ruë tient de la légende. Nicolas Ier vient à Novgorod le 25 juillet et se rend dans une colonie de grenadiers, prodiguant des reproches tant aux colons qu'aux officiers qui n'ont pas su maintenir l'ordre (A. D. Ferguson, art. cit., p. 114 ; Lykošin, art. cit., p. 110)

99. D'après Evstaf'ev, cité par Ferguson (art. cit., p. 114-115), 3600 personnes ont été jugées et punies, parmi lesquelles 129 sont mortes à la suite des châtiments qui leur ont été infligés.

100. Le comte de Witte exerce son autorité sur les colonies du Sud sous les ordres d'Arakčeev jusqu'à la disgrâce de ce dernier en 1826, comme nous l'avons indiqué précédemment (voir note 29) et nous avons vu qu'il ne faut pas exagérer la différence entre les systèmes des colonies de Novgorod et du Midi (voir note 74). Les opinions sur Witte ne sont pas toutes positives. Citons, par exemple, von Bradke qui a travaillé à ses côtés dans les colonies de Nouvelle Russie, et le dépeint en ces termes : "C'était un homme doué d'une grande intelligence, d'une perspicacité rare, mais sans profondeur, d'une éducation étendue mais superficielle, d'un grand tact et capable de savoir à qui il avait à faire au premier coup d'œil. Il ne refusait rien mais ne respectait jamais ses promesses, faisait de l'esprit sur tout mais n'enquêtait sur rien.» («Avtobiografičeskie zapiski ...», art. cit. , t. III, p. 261). Witte jouissait néanmoins d'une grande faveur tant auprès d'Alexandre ${ }^{\mathrm{I} r}$ que de Nicolas I ${ }^{\mathrm{er}}$ (voir rescrit cité le félicitant des résultats obtenus dans les colonies lors de la famine de 1833, note 76).

101. Sur la division de lanciers (uhlans) de Tchougouev, voir note 75. L'établissement de cette colonie s'est heurté, en 1818-1819, à une forte opposition des cosaques du Boug et des autres colons (protestation contre la réquisition des terres, l'abolition de privilèges anciens, refus de couper les foins servant de fourrage pour les chevaux du régiment en 1819) qui n'a été vaincue qu'après de nombreuses arrestations $(1$ 104) et l'instauration de tribunaux militaires devant lesquels sont déférées 313 personnes (voir Petrov, p. 146-151 ; P. P. Evstaf'ev, op. cit., p. 9091 ; et également Karcov, qui donne une version différente de cet épisode, art. cit. , t. 207, p. 89-92). Sur l'établissement de la division de cuirassiers, nous n'avons aucun détail. Mais, pour suivre «le système du comte de Witte », elle doit être postérieure à 1821 , date de sa première mise en œuvre dans les colonies de Kherson.

102. Des rumeurs sur la suppression des colonies militaires courent régulièrement depuis 1'accession au trône de Nicolas I ${ }^{e r}$. La révolte des colonies de Novgorod et leur réorganisation en a suscité de nouvelles. 
subsistance dans le gouvernement de Tamboff, à cause de la disette. Ce déplacement coûte 1400000 roubles.

Les colonies de Kerson consistent en 4 divisions, une de cuirassiers, deux de lanciers et une de houzards récemment admise à la colonisation : ces quatre divisions y compris leurs quarante-huit escadrons de réserve, présentent un effectif de 21120 hommes disponibles, bien montés, bien exercés et dont le recrutement et les remontes sont assurés. Cependant on a été peu satisfait des régiments que l'on a envoyés à l'armée de Turquie, leur tenue était fort belle, mais ils n'avaient point l'esprit ni les habitudes militaires. Ils se sont fondus très vite et ont rendu peu de services ${ }^{103}$.

Dans la campagne de Pologne les troupes colonisées furent peu employées parce que l'on n'était pas sûr de leur esprit ${ }^{104}$. Cependant la Russie était tellement épuisée à l'époque de cette guerre qu'on fit marcher en Pologne une partie des équipages de la flotte de la mer Noire. La colonisation dans le gouvernement de Kerson s'est organisée de la manière suivante: une population composée de Cosaques, de Moldaves, de Valaques, de Serviens et de Tartares, occupait une vaste étendue de steppe entre le Boristhène et le Boug, dans une longueur de 70 lieues de l'est à l'ouest, et le long du Boristhène, de l'Ingoul, de l'Ingouletz, du Boug et de quelques uns de leurs affluents, depuis Novomirgorod jusqu'à Nicolaieff ${ }^{105}$.

Ce ramassis de mauvais sujets pouvait devenir inquiétant un jour. La colonisation les a agglomérés et les a façonnés à un ordre social régulier. Les moyens ont été acerbes, la force a dû être employée, mais le résultat est aujourd'hui satisfaisant ${ }^{106}$.

Une étendue d'environ 2400000 dessetines de terre (environ 3500000 hectares) forme la dotation de la colonie et comme il n'y a point de bois dans ce pays, des forêts du gouvernement de Kiow sont affectées à ses besoins. Une terre vierge, une végétation forte, assurent la subsistance des colons et des troupes.

103. Ce jugement sur les médiocres prestations dans la guerre de Turquie n'est pas corroboré par Ferguson (art. cit., p. 112).

104. Plusieurs divisions de grenadiers cantonnées dans la région de Novgorod ont participé à la répression de la révolution polonaise. Leur absence à l'époque de l'insurrection de ces colonies explique son expansion, car elle ne put être contrée immédiatement par des régiments d'active qui se trouvaient alors engagés en Pologne (P. Karcov, art. cit., t. 208, p. 94). Le général de Witte prit part à la campagne de Pologne à la tête de divisions de cavalerie colonisées (A. D. Ferguson, art. cit., p. 112-113).

105. Cette énumération de peuples est donnée sous la même forme par Tanski, qui cependant emploie le terme «Tatars» et non le traditionnel «Tartares» (op. cit. p. 120). La région concernée, entre le Borysthène (Dniepr) au nord, et le Boug méridional à l'ouest, a bien un peuplement multiethnique. Des Serbes et d'autres ressortissants chrétiens de l'Empire ottoman y défendaient au XVIII' siècle la frontière méridionale (Nouvelle-Serbie). Les cosaques du Boug sont composés de Moldaves, Valaques, Bulgares et autres représentants de peuples danubiens passés au service de la Russie. Sur cette mosaïque ethnique, voir E. I. Družinina, Južnaja Ukraina...,op. cit., p. 71-73,99.

106. Tanski fait suivre son énumération de peuples par ces mots : «qui tous détestent les Russes, et qu'on voulait dompter et réduire » (op. cit., p. 120). Nous avons vu que la colonisation des cosaques du Boug, à Tchougouev, s'est faite en brisant leur opposition. Ce n'est que contraints et forcés, que d'autres cosaques du Boug et des paysans de la Couronne entrent dans les colonies de Kherson. 
87000 âmes mâles se sont trouvées habiter cette portion du gouvernement ${ }^{107}$. Ils ont été distraits de l'autorité civile à laquelle ils étaient soumis et ont été affectés à la subsistance et au recrutement des seize régiments de cavalerie. Elisabethgrad qui est le quartier général est le seul sous l'administration civile ${ }^{108}$.

Deux modes de colonisation ont été suivis : en 1818 la division de lanciers du Boug est entrée en cantonnement dans le lieu qui lui était affecté à Vosnasensk ${ }^{109}$, et l'on a construit, défriché, en un mot tout établi en sa présence. 18 bataillons d'infanterie y étaient occupés et il a fallu nourrir habitants et troupes jusqu'à ce qu'on ait obtenu des récoltes suffisantes pour subvenir aux besoins de tout ce monde. On a senti le vice de ce mode et la division d'Ukraine n'a envoyé que des cadres pour les escadrons de réserve et de cantonnistes à Novomirgorod ${ }^{110}$, c'est-àdire vingt-sept officiers et soixante sous-officiers par régiment. On a alors cadastré le pays, divisé la population, établi les écoles, les haras, fait des magasins de réserve, façonné aux exercices militaires ceux qui devaient être soumis à la discipline et lorsque la production a été suffisante, en 1821, les régiments sont arrivés. Toutes les constructions n'étaient pas encore achevées et on a reconnu qu'on pouvait mieux faire.

La division des cuirassiers dont le quartier général est à Petrikoska111, n'est entrée qu'en haut de quatre ans de préparation dans ses établissements, au mois d'avril 1826, et lorsque hommes, chevaux et subsistances ont été en état et toutes les constructions achevées ${ }^{112}$. Ici il n'y a point de luxe dans les bâtisses. Il n'y a de

107. La superficie de 2400000 déciatines correspond à l'ensemble des colonies en 1825 , et non aux seules colonies de Nouvelle Russie (voir I. F. Dubrovin, « Materialy i čerty k biografii imperatora Nikolaja I... », art. cit., p. 419). L'expansion des terres colonisées s'est certes poursuivie dans les premières années du règne de Nicolas ${ }^{\mathrm{er}}$ mais elle ne touche d'immenses territoires qu'à la fin de 1837, par la colonisation de terres confisquées aux insurgés polonais dans les gouvernements de Kiev et de Podolsk (voir A. D. Ferguson, art. cit., p. 111, 120). Pour les colonies de cavalerie de Nouvelle Russie, Družinina donne le chiffre de 681119 déciatines en 1822 (E. I. Družinina, op. cit., p. 100). Il y a eu certes de nouveaux établissements depuis, mais le chiffre avancé par La Ruë semble surévalué. Quant à la population menant des travaux agricoles sur ces immenses domaines, von Bradke l'évalue bien aussi à 90000 âmes.

108. Les données officielles classent Elizavetgrad parmi les «villes militaires» (I. F. Dubrovin, art. cit., p. 420).

109. Voznessensk, ville du gouvernement de Kherson, dans le distirict d'Elizavetgrad, sur le Boug. À l'époque des cosaques zaporogues, elle portait le nom de Sokoli.

110. Novomirgorod, ville du gouvernement de Kherson dans les district d'Elizavetgrad, sur le Grand Vysa. Elle a été le centre de la Nouvelle Serbie et conserve une certaine importance commerciale.

111. Petrikoska, faubourg du district d'Aleksandriia dans le gouvernement de Kherson, rebaptisé Novaia Praga, en raison de la conduite brillante du régiment qui y était colonisé lors de la campagne de Pologne. Celui-ci, traversant les lignes polonaises, arriva aux portes de Prague (Marmont, Voyage du maréchal, duc de Raguse ..., op. cit., p. 260-261).

112. Il n'est pas facile de suivre dans le temps et dans l'espace l'établissement de ces diverses colonies et des régiments qui leur sont affectés, ces derniers changeant de nom (les anciens régiments de cosaques d'Ukraine prennent le nom d'uhlans du Boug et d'Ukraine et incorporent dans leurs rangs d'anciens cosaques du Boug). L'un des intérêts du rapport de La Ruë est de montrer que chaque établissement se fait suivant un processus différent. C'est pour le cantonnement des cuirassiers lancé à la fin de 1821 qu'est expérimenté le «nouveau système » de Witte consistant, dès la création de la colonie, à faire nourrir par les 6000 paysans de la 
régulier que les établissements publiques. Les habitants ont conservé leurs maisons, seulement on leur donne des modèles et des facilités pour en construire de meilleures et on les oblige à se rapprocher les uns des autres. Ainsi les villages prennent des quarts, des demis, des escadrons entiers.

Autant que possible on laisse à chacun le terrain qu'il cultivait, seulement on l'a augmenté jusqu'à concurrence de quinze dessetines (environ vingt-deux hectares) à ceux qui en avaient moins, quantité qui a été reconnue nécessaire pour la subsistance d'une famille coloniale.

Neuf à dix mille dessetines ont été affectées à 1a consommation des haras et à peu près une pareille quantité de terre labourable et cultivée au moyen de quarante journées de corvée par tenance pour fournir l'avoine nécessaire à la consommation journalière et entretenir les magasins de réserve en grains de tous genres dans le cas d'une année malheureuse. Enfin des prairies qui sont fauchées par les habitants et par la troupe approvisionnent les magasins de la cavalerie.

Sous le rapport de l'agriculture on voit en parcourant le pays que l'état des colons est satisfaisant ${ }^{113}$. Non seulement la consommation est assurée, mais on a pu venir au secours de la population des gouvernements voisins pendant cette année de disette et après avoir distribué des secours à 400000 âmes de population et 450000 têtes de bétail, ainsi que je l'ai signalé dans le rapport sur l'état du gouvernement du midi, il restait encore au mois de mai cette année 200000 tchetwerts de grains, c'est-à-dire dix-huit mois d'approvisionnement pour la troupe.

Les moyens de recrutement présentent un excédent considérable, plusieurs régiments comptaient 1700 hommes dans 1a réserve, on a senti la nécessité d'en diminuer le nombre et pour rapprocher le plus possible les charges des habitants composant les colonies militaires des autres sujets russes. Les réserves des régiments ont été diminuées et les écoles ne sont plus suivies que par 50 enfants dans chaque escadron colonisé, c'est-à-dire par le nombre d'enfants représentant celui que le recrutement annuel de tout l'empire appelle d'hommes sous les drapeaux en proportion

Couronne qui y sont enrôlés les cadres (400 personnes) venus l'organiser et à obtenir d'eux dans un délai de trois ans l'approvisionnement de toute la division et le fourrage pour les chevaux (Petrov, p. 174-176 ; P. Karcov, art. cit., t. 207, p. 95).

113. Si Raguse vante la prospérité des colons de Voznessensk (Marmont, Voyage du maréchal, duc de Raguse ..., op. cit., p. 237), von Bradke fait au contraire une critique sévère d'une agriculture menée sans règles et qui épuise le sol pour de longues périodes (de cinq ans à plusieurs dizaines d'années). Il a pour mission de rendre l'exploitation des terres plus rationnelle et décrit la situation qu'il trouve à son arrivée (entre 1826 et 1831). Il dénonce les ensemencements faits sans considération du nombre de bras nécessaires à la récolte, et, sur les lopins des colons, les céréales grillant sur pied sous le soleil, car ne pouvant pas être récoltées à temps. Parlant de la situation désespérée des colons, il fait édicter par le comte de Witte l'ordre de ne les faire travailler pour l'État que trois jours par semaine ce qui, dit-il, réduit l'arbitraire auquel ils étaient soumis («Avtobiografičeskie zapiski ... », art. cit., t. III, p. 258-259). La misère des colons est aussi constatée par Arakčeev lors de sa tournée d'inspection de 1821 dans les colonies de Kherson (Petrov, p. 169-170). On pourrait penser que les problèmes initiaux ont été réglés, ce qui expliquerait l'apparente prospérité régnant au milieu des années 1830. Mais, le rapport dressé en 1856 sur les colonies du Midi fait, lui aussi, état de la pauvreté des colons, dont une grande partie ne disposerait d'aucune bête de somme (D. Stolypin, « Ob uprazdnenii voennyh poselenij » (À propos de la suppression des colonies militaires), Russkij arhiv, t. III, 1874, col. 767-769. 
de la population ${ }^{114}$. Ainsi le système des colonies militaires en ce qui concerne le recrutement a maintenant pour objet l'éducation des enfants destinés à être soldats un jour ; et quant à l'économie, de faire vivre à meilleur marché les soldats des escadrons actifs cantonnés chez les paysans plus spécialement destinés à recruter ces mêmes régiments. L'espèce d'hommes est superbe.

La composition des régiments colonisés est la même que celle des régiments ordinaires quant aux escadrons de guerre, mais ces corps ont trois escadrons de réserve et un de cantonnistes. 27 officiers et 60 sous officiers en composent les cadres ; néanmoins ce serait à tort que l'on ferait compter cette partie de la colonisation dans l'effectif de l'armée. Ce ne sont au fait que des moyens d'alimenter et de recruter les escadrons de guerre.

Les escadrons de réserve n'ont que 100 chevaux chacun dont 50 sont de réforme et destinés à l'instruction des cantonnistes. En hommes ils sont nombreux. L'escadron des cantonnistes est composé de 200 jeunes gens de 14 à 18 ans ${ }^{115}$. C'est une véritable école militaire d'où il sort des cavaliers tout formés qui attendent ensuite dans les escadrons de réserve que les besoins du service les appellent dans les rangs des escadrons réellement disponibles.

Dans tous les cas les cadres de la colonie en officiers et en sous-officiers ne doivent jamais en sortir, leur position étant toute d'administration ou d'instruction.

Quant aux moyens de remonte, la quantité de poulains m'a paru considérable, les étalons beaux et les juments bien choisies. Le succès de ces haras de cavalerie est évident. On a formé un haras par régiment, chaque haras est composé de trois cent quarante-cinq juments et de vingt-trois étalons dans les cuirassiers et de vingtsept dans les lanciers. Les étalons des haras de cuirassiers sont des hunters anglais, d'autres viennent des beaux haras russes de la comtesse Orloff, du comte Zawadasski et du prince Repnin. Les étalons des lanciers viennent des haras du prince Sanguskoi en Volhynie, d'autres d'Ukraine et des steppes.

Le prix moyen des étalons de cuirassiers est de 5000 roubles quoique le gouvernement n'en alloue que 2500. Celui des haras de lanciers de 2500 à 3000 roubles, le gouvernement n'allouant que $1500^{116}$. Cette augmentation provient de ce qu'on a employé une partie de l'argent destiné à l'acquisition des juments, celles-ci ayant

114. Ce rapprochement des statuts des paysans de la Couronne, des soldats-laboureurs, des colons et des troupes irrégulières est bien, comme nous l'avons vu, l'une des grandes lignes des réformes de 1829-1835.

115. Les fils de colons, nommés cantonistes, sont répartis en trois classes d'âge : jusqu'à 7 ans, de 7 à 14 ans (initialement 12), de 14 (initialement 12) à 18 ans. Les deux premières catégories résident chez leurs parents, la troisième dans un internat créé dans chaque compagnie (voir art. cit., Le Spectateur militaire, IX, 1830, p. 478-480).

116. Tanski donne des chiffres semblables, mais en francs et non en roubles : pour les étalons des cuirassiers dans les haras du comte Orlof, du comte Zavadowski ou du prince Repnin (jusqu'à 5000 fr., l'allocation du gouvernement étant de 2500 fr.) et pour les étalons des uhlans tirés des haras du prince Sanguszko (3 000 fr., le double de l'allocation du gouvernement) (op. cit., p. 131-132). Les haras des colonies, entraînant des frais considérables, ont été supprimés en 1844 (I. F. Dubrovin, art. cit., p. 422). 
été tirées en grande partie des régiments, ce qui fait qu'elles n'ont coûté que le prix de remonte. D'autres ont été acquises à bon marché dans les steppes.

Les hunters anglais ont été croisés avec les juments de l'Ukraine, ce qui a donné une espèce de chevaux pleins de force, de membres et de souplesse pour les cuirassiers.

Pour la cavalerie légère, on a croisé des étalons persans avec les juments du Don et des steppes, dont les produits font une excellente espèce de chevaux pour les lanciers et les houzards.

Les officiers des régiments colonisés recevaient une augmentation de traitement pour tenir lieu des abus administratifs dont ils profitaient ${ }^{117}$. Ils ne reçoivent plus cette année cette augmentation par suite des dépenses qu'a dû supporter l'état, occasionnées par la disette, et leur mécontentement s'en est accru. Leur position actuelle n'est plus bien définie. De tous temps ce régime leur déplaisait et depuis la suppression des colonies d'infanterie, depuis les concessions qui rapprochent l'état des troupes colonisées des autres troupes, il est évident à tous les yeux que les officiers répugnent un peu plus encore à ce système, qu'ils s'en exagèrent peut-être même les ennuis et les inconvénients pour eux, et qu'ils en souhaitent la suppression $^{118}$. En effet leur position est en ce moment plus défavorable que celle des autres troupes. Ils supportent encore une partie des exigences du système colonial, sans y trouver les avantages et les économies qu'ils avaient droit d'attendre de ce régime. Aussi l'on avait avant pourvu à leurs moyens de nourriture, de logement, d'ameublement et de remonte. Aujourd'hui ils pourvoyent à toutes les nécessités comme ils l'entendent, seulement on leur donne quelques facilités tirées des ressources de la colonie, par exemple ils peuvent acheter des meubles à extrêmement bon marché, faits par les hommes du bataillon d'ouvriers attachés aux colonies, mais ils se considèrent tellement comme dans une position transitoire, que tous ceux que j'ai vus chez eux, n'y étaient que campés ; très peu sont mariés.

A en juger par aperçu, le système des colonies de cavalerie doit présenter des économies au gouvernement, d'une part la nourriture des hommes et des chevaux, les remontes et une partie des frais divers présentent une diminution de dépenses de 240000 roubles par régiment. Mais aussi il y a 27 officiers et 60 sous-officiers formant les cadres des escadrons de colons qui sont à payer.

117. L'auteur de l'article dans Le Spectateur militaire de 1830 affirme que les officiers, deux ans après leur entrée dans le district de la colonie, reçoivent une augmentation de solde de la moitié de leur traitement (p. 482). Pour Karcov, une telle augmentation est réservée aux commandants dont le régiment obtient sa subsistance des colons (art. cit., t. 207, p. 95). Enfin, Petrov explique l'intéressement personnel de l'encadrement militaire à obtenir ce résultat et les abus que ce système engendre (p. 178-179).

118. Le mécontentement des officiers des colonies de Novgorod, surtout à l'époque d'Arakčeev, ressort des Mémoires écrits par plusieurs d'entre eux. Ceux de A. K. Gribbe expliquent la condition des officiers, la rudesse des relations entre supérieurs et subalternes, les punitions infligées pour des vétilles, l'implacable discipline rendant toute expression du mécontentement impossible et leurs attentes après l'avènement de Nicolas I ${ }^{\text {er }}$ ( Novgorodskie voennye poselenija... », art. cit., p. 127-152). Le témoignage de La Ruë sur l'état d'esprit des officiers dans les colonies du Sud en 1834 est d'autant plus intéressant que nous avons peu de documents sur le sujet. 
Enfin il faut faire entrer dans la balance ce que la population payait au gouvernement. Néanmoins il y a en résumé tout lieu de croire cet excédant de dépenses compensé, qu'un régiment de cavalerie colonisé doit coûter 120000 roubles de moins par an que celui qui ne l'est pas ${ }^{119}$. Il est vrai qu'il y a une première mise de fonds pour les constructions qui a été considérable. L'évaluation en est difficile à faire et le gouvernement lui-même doit avoir de la peine à s'en rendre compte, parce qu'il ne retirait aucun revenu des bois employés, parce que la main d'œuvre ayant été faite par des soldats, une augmentation de solde de dix copecks par jour est un fort petit objet; enfin parce que la caisse des colonies a pourvu à beaucoup de ces dépenses au moyen des frais de nourriture des hommes et des chevaux des cadres qu'on a fait nourrir par les habitants pendant plusieurs années, et une grande partie des constructions étant d'une utilité civile plutôt que militaire, ne peut être exclusivement attribuée à l'établissement des colonies militaires.

Les subsistances, le recrutement, les remontes étant assurés, ainsi que le résultat économique, il paraît que le but militaire des colonies est en grande partie atteint. On ne peut assurer d'une manière aussi positive que les abus administratifs et les vexations locales ayant disparu, les habitants ont de l'aisance, et cependant sont malheureux $^{120}$. Les soldats ne paraissent point gais ni satisfaits, et les officiers ne cachent point leur mécontentement. Est-ce l'effet pour la population d'une transition trop brusque de l'état sauvage à une civilisation improvisée ${ }^{121}$ ? Ce qu'il y a de certain c'est qu'on ne peut prévoir l'influence politique que ce système aura indubitablement sur l'avenir du pays.

Une population toute entière instruite, qui n'est pas propriétaire et qui en supporte toutes les charges, à laquelle on donne des armes, des chefs mécontents, peut certainement dans des circonstances difficiles devenir dangereuse pour la tranquillité de l'État. Les colonies d'infanterie établies dans le nord l'ont prouvé, quoique leur mouvement insurrectionnel n'eut aucune intention politique ${ }^{122}$. Mais dès aujourd'hui on peut affirmer que la Russie a en Ukraine une place d'armes contre la Turquie qui est en même temps très menaçante contre l'Autriche.

119. Cette estimation est proche de celle de Petrov. Pour lui, lorsque la subsistance est assurée par la colonie, l'État économise 100000 roubles par régiment de cavalerie et 80000 par régiment d'infanterie (p. 176).

120. Voir note 32 sur le malheur des habitants. Voir également Tanski qui dresse un tableau très noir de la situation des colons (op. cit., p. 145-147).

121. Sur ce passage à la civilisation, unanimement perçu par les auteurs occidentaux de l'époque, se reporter à la présentation des rapports (p. 530).

122. Plusieurs rapports signalent à Nicolas Ier le danger que représentent les colonies militaires pour la sécurité de l'État : le rapport établi à la demande de l'empereur en 1826, au lendemain de l'insurrection décabriste, alors qu'il semble que les officiers insurgés avaient eu l'intention de provoquer des troubles dans les colonies (voir Petrov, p. 193-198) ; le rapport de Benkendorv de l'été 1831, mentionnant la persistance de l'agitation des partisans des décabristes en Pologne et dans les colonies militaires (cité par A. D. Ferguson, art. cit., p. 115). 


\section{Cavalerie colonisée}

\begin{tabular}{|c|c|c|c|c|c|c|}
\hline \multirow[b]{2}{*}{ Armes } & \multicolumn{2}{|c|}{ Divisions } & \multirow[b]{2}{*}{ Régiments } & \multirow{2}{*}{$\begin{array}{l}\text { Escadrons } \\
\text { actifs }\end{array}$} & \multirow{2}{*}{$\begin{array}{l}\text { Escadrons } \\
\text { de réserve }\end{array}$} & \multirow[b]{2}{*}{ Observations } \\
\hline & $\begin{array}{c}\text { de } \\
\text { cuirassiers }\end{array}$ & légères & & & & \\
\hline Cuirassiers & 2 & - & 8 & 64 & 24 & $\begin{array}{c}\text { Une division à } \\
\text { Tchougouieff et } \\
\text { une autre dans le } \\
\text { gouvernement de } \\
\text { Kerson }\end{array}$ \\
\hline Lanciers & - & 3 & 12 & 96 & 36 & $\begin{array}{c}\text { Une division à } \\
\text { Tchougouieff et } \\
\text { deux dans le } \\
\text { gouvernement } \\
\text { de Kerson }\end{array}$ \\
\hline Hussards & - & 1 & 4 & 32 & 12 & $\begin{array}{c}\text { Dans le } \\
\text { gouvernement } \\
\text { de Kerson }\end{array}$ \\
\hline Total & 2 & 4 & 24 & 192 & 72 & \\
\hline
\end{tabular}

Ces 6 divisions, en calculant l'escadron à 120 chevaux, présentent donc un effectif de 31680 hommes montés y compris les 72 escadrons de réserve qui sont toujours au complet dans les colonies. Il serait trop long de donner dans ce rapport une idée complète du système de la colonisation. C'est un ordre social tout entier. Quatorze volumes de règlements les régissent: les obligations des soldats colonisés, des chefs colons, leurs droits, leurs devoirs y sont tracés. Les mesures de précaution pour assurer les greniers d'abondance, et une réserve en argent, qui fait le service d'une banque d'emprunt n'y ont point été oubliés. Le sort des invalides, l'éducation des enfants des différents sexes et âges y sont prescrits. Le mode de tester et de succéder est réglé et une sorte de municipalité élective est établie au milieu de ce despotisme militaire ${ }^{123}$.

Dans le rapport sur la situation des troupes employées au Caucase et sur la ligne, je donne à votre Excellence le détail d'un plan de colonisation militaire qui va être mis en exécution sur l'Araxe ${ }^{124}$.

Je suis avec un profond respect, Monsieur le Maréchal, de votre Excellence le très humble et obéissant serviteur.

Le baron de La Rue

Etc.., etc.

123. Différents comités assurent l'administration locale au niveau du régiment (puis du district, okrug après la réforme de 1832) et au niveau de la compagnie (puis du canton, volost' après 1832). C'est à ce dernier échelon que sont représentés les paysans (voir Le Spectateur miliaire, 1830, art. cit., p. 486).

124. Le projet de créer des colonies militaires le long de la frontière avec la Perse date de 1826. Il n'est mis en œuvre qu'à partir de 1837, et dans des proportions modestes. Ces colons militaires sont reversés dans les régiments cosaques du Caucase au fur et à mesure que progresse la pacification (A. D. Ferguson, art. cit., p. 111, 120 ; Lykošin, art. cit., p. 611). 